Network Working Group

Request for Comments: 2151

FYI : 30

Obsoletes: RFC 1739

Category: Informational
G. Kessler

S. Shepard

Hill Associates, Inc.

June 1997

\title{
A Primer On Internet and TCP/IP Tools and Utilities
}

Status of this Memo

This memo provides information for the Internet community. This memo does not specify an Internet standard of any kind. Distribution of this memo is unlimited.

\section{Abstract}

This memo is an introductory guide to many of the most commonlyavailable TCP/IP and Internet tools and utilities. It also describes discussion lists accessible from the Internet, ways to obtain Internet and TCP/IP documents, and some resources that help users weave their way through the Internet.

Table of Contents

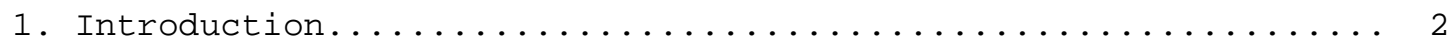

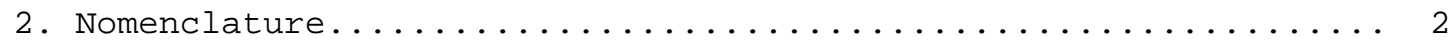

3. Finding Information About Internet Hosts and Domains........ 3

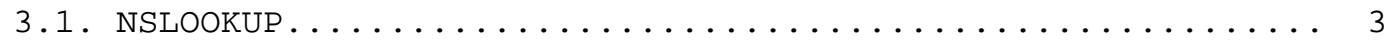

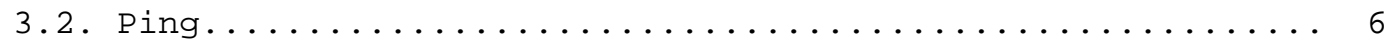

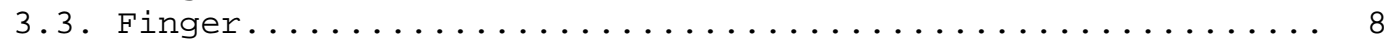

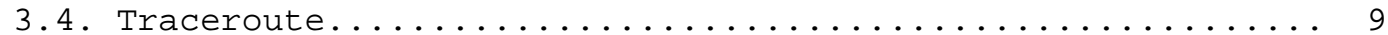

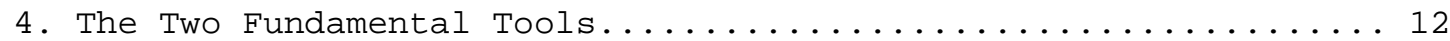

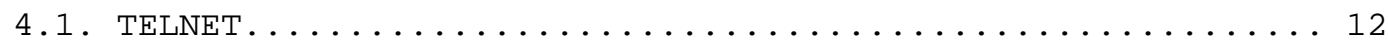

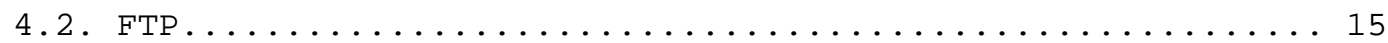

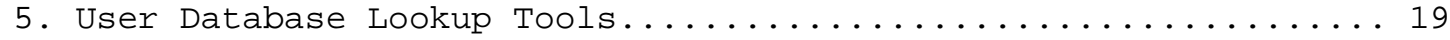

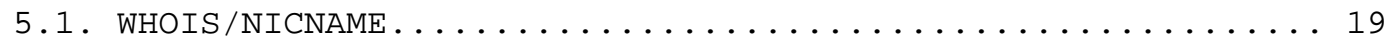

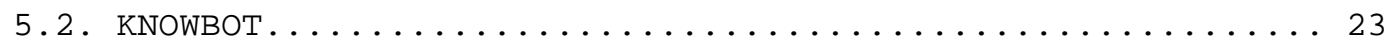

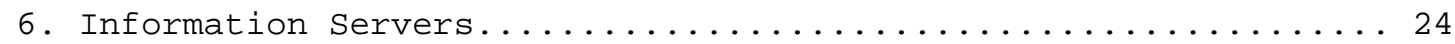

6.1 Archie................................ 24

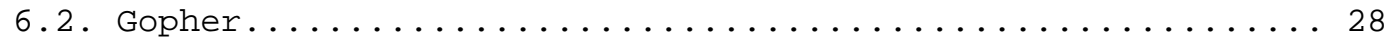

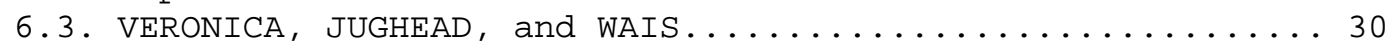

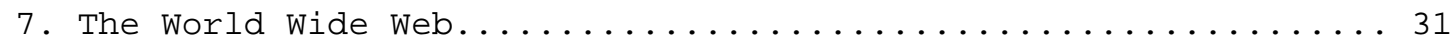

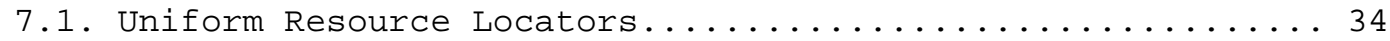

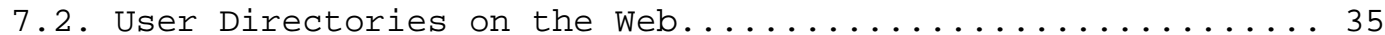

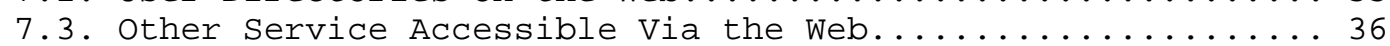

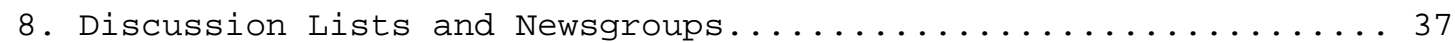

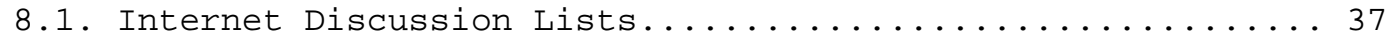




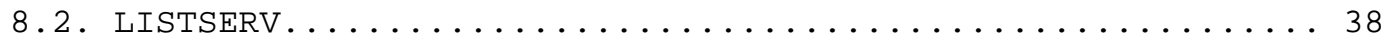

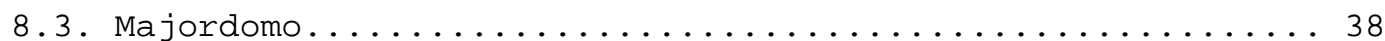

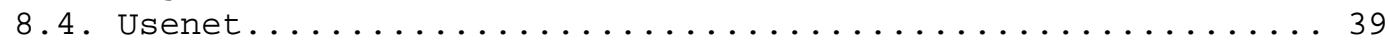

8.5 Finding Discussion Lists and Newsgroups.............. 40

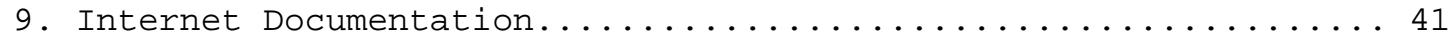

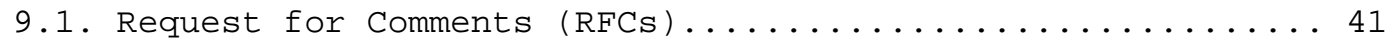

9.2. Internet Standards.......................... 44

9.3. For Your Information Documents................... 45

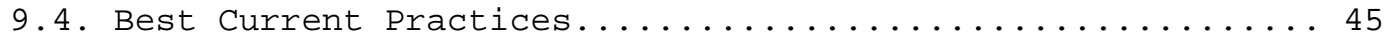

9.5. RARE Technical Reports....................... 46

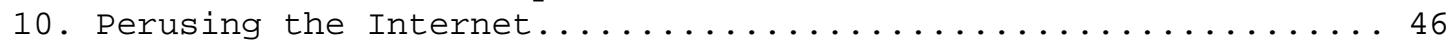

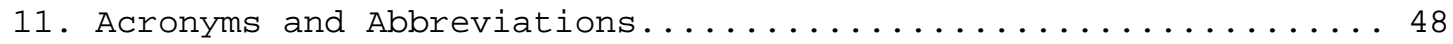

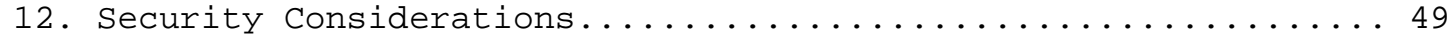

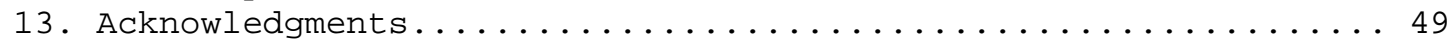

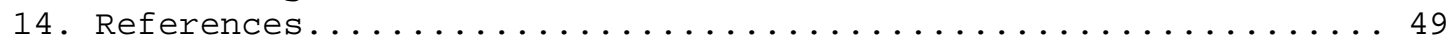

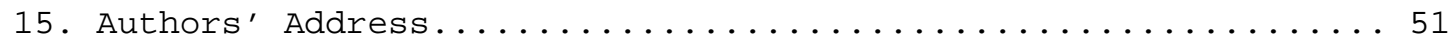

1. Introduction

This memo is an introductory guide to some of the most commonlyavailable TCP/IP and Internet tools and utilities that allow users to access the wide variety of information on the network, from determining if a particular host is up to viewing a multimedia thesis on foreign policy. It also describes discussion lists accessible from the Internet, ways to obtain Internet and TCP/IP documents, and some resources that help users weave their way through the Internet. This memo may be used as a tutorial for individual self-learning, a stepby-step laboratory manual for a course, or as the basis for a site's users manual. It is intended as a basic guide only and will refer to other sources for more detailed information.

\section{Nomenclature}

The following sections provide descriptions and detailed examples of several TCP/IP utilities and applications, including the reproduction of actual sessions using these utilities (with some extraneous information removed). Each section describes a single TCP/IP-based tool, it's application, and, in some cases, how it works. The text description is usually followed by an actual sample session.

The sample dialogues shown below were obtained from a variety of software and hardware systems, including AIX running on an IBM RS/6000, Linux on an Intel 486, Multinet TCP/IP over VMS on a VAX, and FTP Software's OnNet (formerly PC/TCP) running on a DOS/Windows PC. While the examples below can be used as a guide to using and learning about the capabilities of TCP/IP tools, the reader should understand that not all of these utilities may be found at all TCP/IP hosts nor in all commercial software packages. Furthermore, the user 
interface for different packages will be different and the actual command line may appear differently than shown here; this will be particularly true for graphical user interfaces running over Windows, $\mathrm{X}$-Windows, OS/2, or Macintosh systems. Windows-based sessions are not shown in this RFC because of the desire to have a text version of this document; in addition, most GUI-based TCP/IP packages obscure some of the detail that is essential for understanding what is really happening when you click on a button or drag a file. The Internet has many exciting things to offer but standardized interfaces to the protocols is not yet one of them! This guide will not provide any detail or motivation about the Internet Protocol Suite; more information about the TCP/IP protocols and related issues may be found in RFC 1180 [29], Comer [6], Feit [7], Kessler [14], and Stevens [30].

In the descriptions below, commands are shown in a courier font (Postscript and HTML versions); items appearing in square brackets ([]) are optional, the vertical-bar (|) means "or," parameters appearing with no brackets or within curly brackets ( $\{$ ) are mandatory, and parameter names that need to be replaced with a specific value will be shown in italics (Postscript and HTML versions) or within angle brackets ( $<>$, text version). In the sample dialogues, user input is in bold (Postscript and HTML versions) or denoted with asterisks $(* *)$ in the margin (text version).

3. Finding Information About Internet Hosts and Domains

There are several tools that let you learn information about Internet hosts and domains. These tools provide the ability for an application or a user to perform host name/address reconciliation (NSLOOKUP), determine whether another host is up and available (PING), learn about another host's users (Finger), and learn the route that packets will take to another host (Traceroute).

\subsection{NSLOOKUP}

NSLOOKUP is the name server lookup program that comes with many TCP/IP software packages. A user can use NSLOOKUP to examine entries in the Domain Name System (DNS) database that pertain to a particular host or domain; one common use is to determine a host system's IP address from its name or the host's name from its IP address. The general form of the command to make a single query is:

$$
\text { nslookup [IP_address|host_name] }
$$

If the program is started without any parameters, the user will be prompted for input; the user can enter either an IP address or host name at that time, and the program will respond with the name and 
address of the default name sever, the name server actually used to resolve each request, and the IP address and host name that was queried. Exit is used to quit the NSLOOKUP application.

Three simple queries are shown in the example below:

1 Requests the address of the host named www.hill.com, the World Wide Web server at Hill Associates. As it turns out, this is not the true name of the host, but an alias. The full name of the host and the IP address are listed by NSLOOKUP.

2 Requests the address of host syrup.hill.com, which is the same host as in the first query. Note that NSLOOKUP provides a "nonauthoritative" answer. Since NSLOOKUP just queried this same address, the information is still in its cache memory. Rather than send additional messages to the name server, the answer is one that it remembers from before; the server didn't look up the information again, however, so it is not guaranteed to still be accurate (because the information might have changed within the last few milliseconds!).

3 Requests the name of the host with the given IP address. The result points to the Internet gateway to Australia, munnari.oz.au.

One additional query is shown in the dialogue below. NSLOOKUP examines information that is stored by the DNS. The default NSLOOKUP queries examine basic address records (called "A records") to reconcile the host name and IP address, although other information is also available. In the final query below, for example, the user wants to know where electronic mail addressed to the hill.com domain actually gets delivered, since hill.com is not the true name of an actual host. This is accomplished by changing the query type to look for mail exchange (MX) records by issuing a set type command (which must be in lower case). The query shows that mail addressed to hill.com is actually sent to a mail server called mail.hill.com. If that system is not available, mail delivery will be attempted to first mailme.hill.com and then to netcomsv.netcom.com; the order of these attempts is controlled by the "preference" value. This query also returns the name of the domain's name servers and all associated IP addresses.

The DNS is beyond the scope of this introduction, although more information about the concepts and structure of the DNS can be found in STD 13/RFC 1034 [19], RFC 1591 [21], and Kessler [16]. The help command can be issued at the program prompt for information about NSLOOKUP's more advanced commands. 
TECHNICAL NOTE: There are other tools that might be available on your system or with your software for examining the DNS. Alternatives to NSLOOKUP include HOST and DIG.

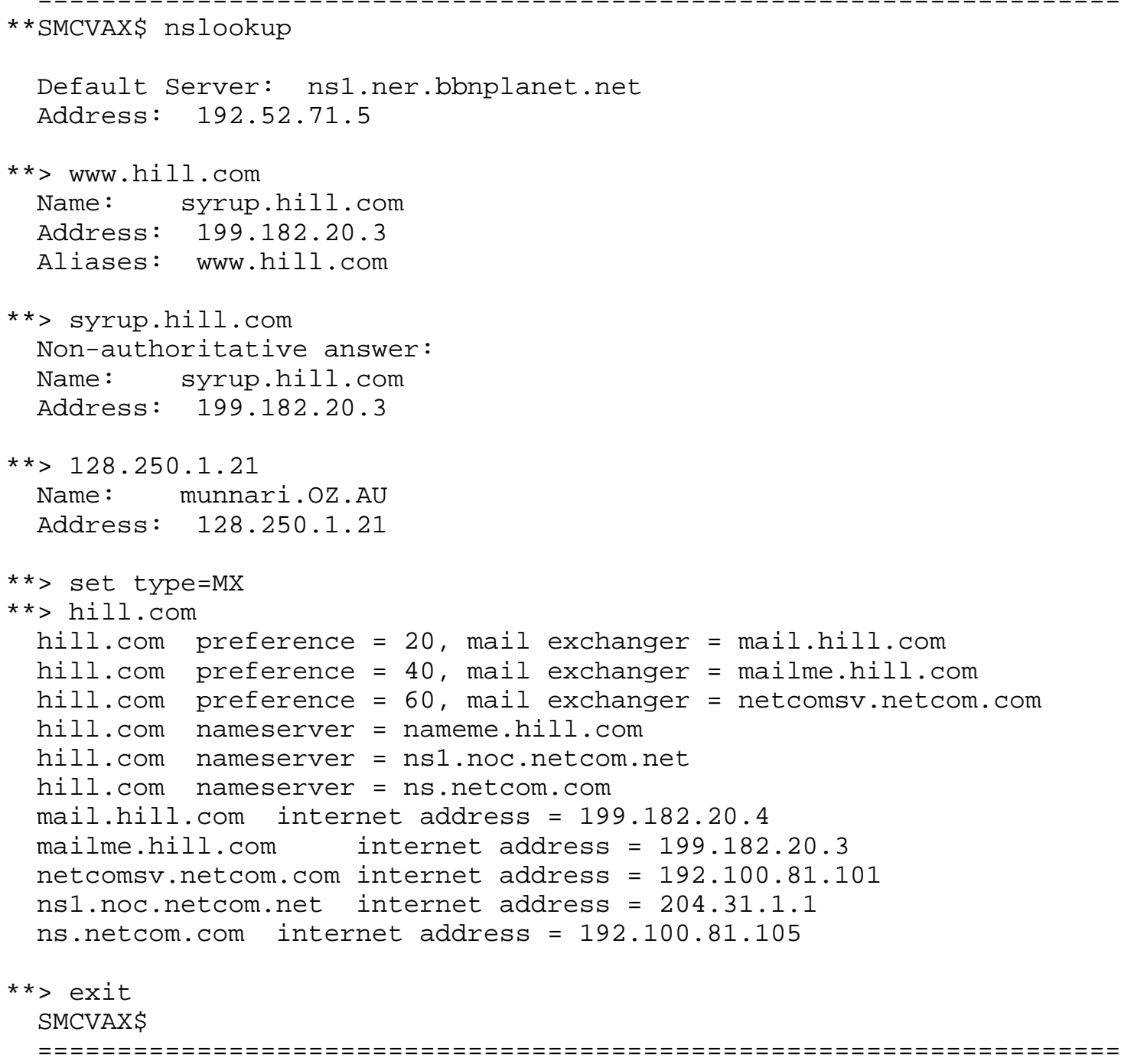




\subsection{Ping}

Ping, reportedly an acronym for the Packet Internetwork Groper, is one of the most widely available tools bundled with TCP/IP software packages. Ping uses a series of Internet Control Message Protocol (ICMP) [22] Echo messages to determine if a remote host is active or inactive, and to determine the round-trip delay in communicating with it.

A common form of the Ping command, showing some of the more commonly available options that are of use to general users, is:

ping $[-\mathrm{q}][-\mathrm{v}][-\mathrm{R}][-\mathrm{C}$ Count] [-i Wait] [-s PacketSize] Host

where:

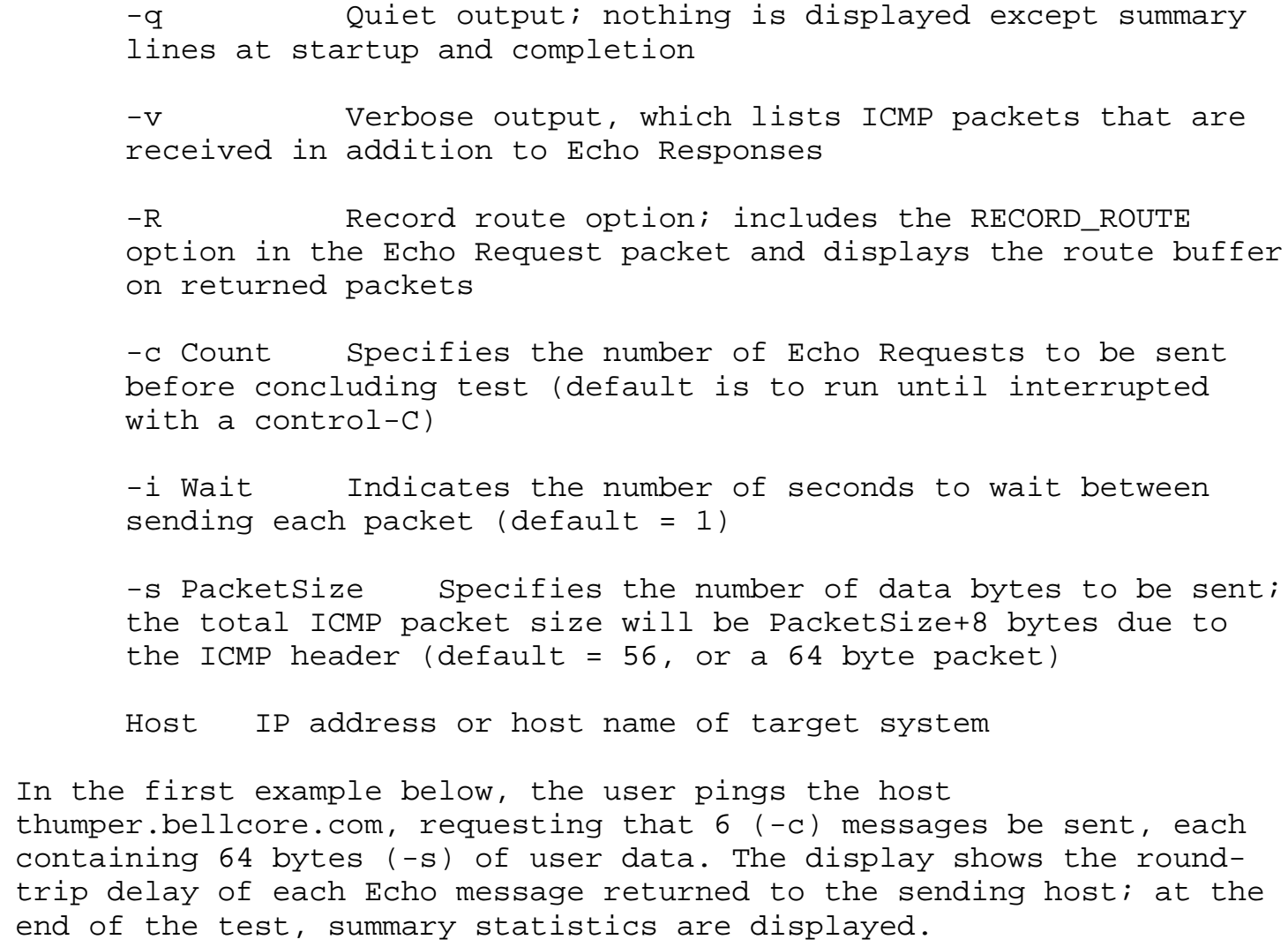


In the second example, the user pings the host smcvax.smcvt.edu, requesting that 10 messages be sent in quite mode (-q). In this case, a summary is printed at the conclusion of the test and individual responses are not listed.

TECHNICAL NOTE: Older versions of the Ping command, which are still available on some systems, had the following general format:

ping $[-\mathrm{S}] \quad\left\{\mathrm{IP} \_\right.$address|host_name $\}$[PacketSize] [Count]

In this form, the optional "-s" string tells the system to continually send an ICMP Echo message every second; the optional Packetsize parameter specifies the number of bytes in the Echo message (the message will contain Packetsize-8 bytes of data; the default is 56 bytes of data and a 64 byte message); and the optional Count parameter indicates the number of Echo messages to send before concluding the test (the default is to run the test continuously until interrupted).

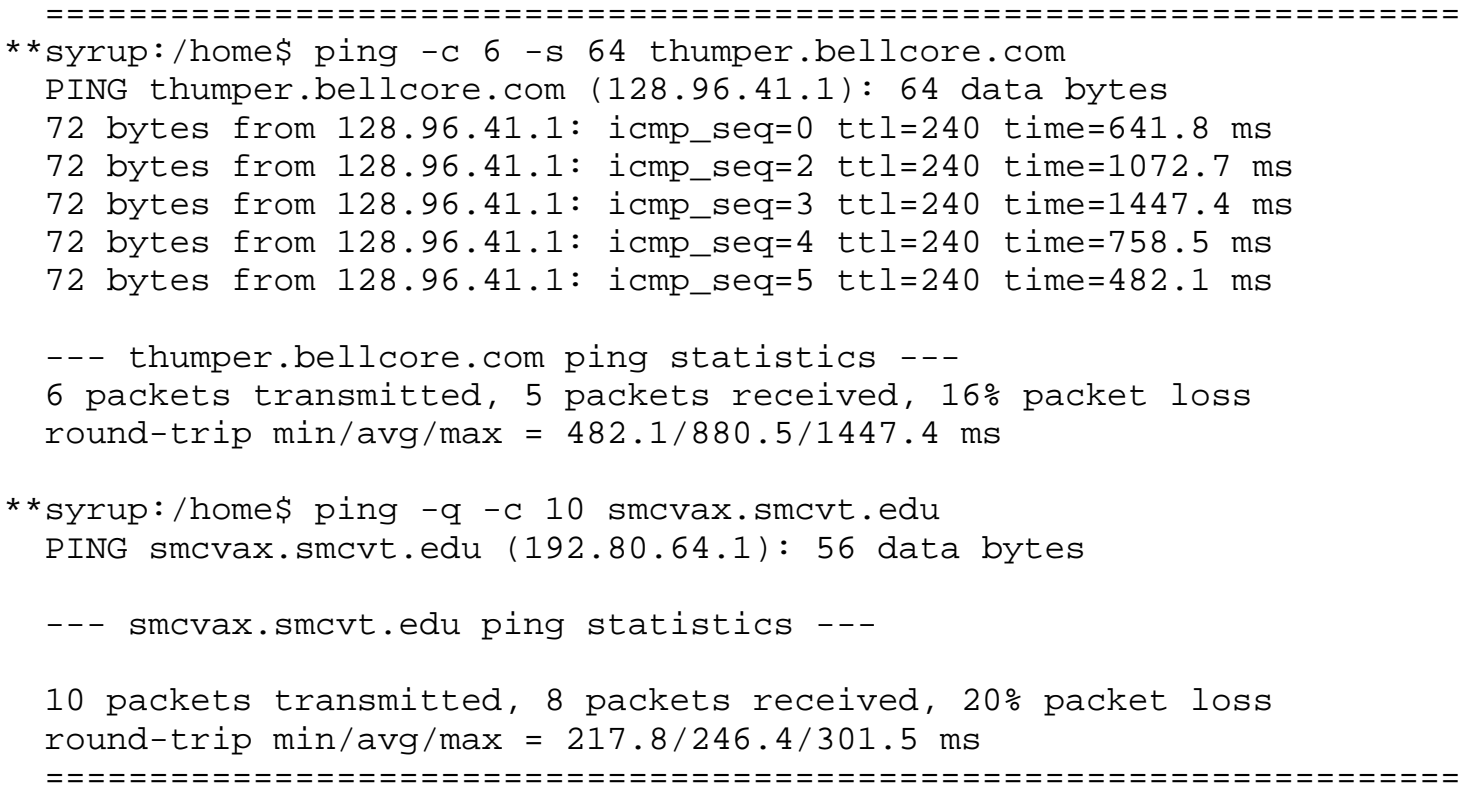




\subsection{Finger}

The Finger program may be used to find out who is logged in on another system or to find out detailed information about a specific user. This command has also introduced a brand new verb; fingering someone on the Internet is not necessarily a rude thing to do! The Finger User Information Protocol is described in RFC 1288 [32]. The most general format of the Finger command is:

finger [username] @host_name

The first example below shows the result of fingering an individual user at a remote system. The first line of the response shows the username, the user's real name, their process identifier, application, and terminal port number. Additional information may be supplied at the option of the user in "plan" and/or "project" files that they supply; these files are often named PLAN.TXT or PROJECT.TXT, respectively, and reside in a user's root directory (or somewhere in an appropriate search path).

The second example shows the result of fingering a remote system. This lists all of the processes currently running at the fingered system or other information, depending upon how the remote system's administrator set up the system to respond to the Finger command.

$\star \star C:>$ finger kumquatesmcvax.smcvt.edu

[smcvax.smcvt.edu]

KUMQUAT Gary Kessler KUMQUAT not logged in

Last login Fri 16-Sep-1996 3:47PM-EDT

Plan:

Gary C. Kessler

Adjunct Faculty Member, Graduate College

INTERNET: kumquatesmcvt . edu

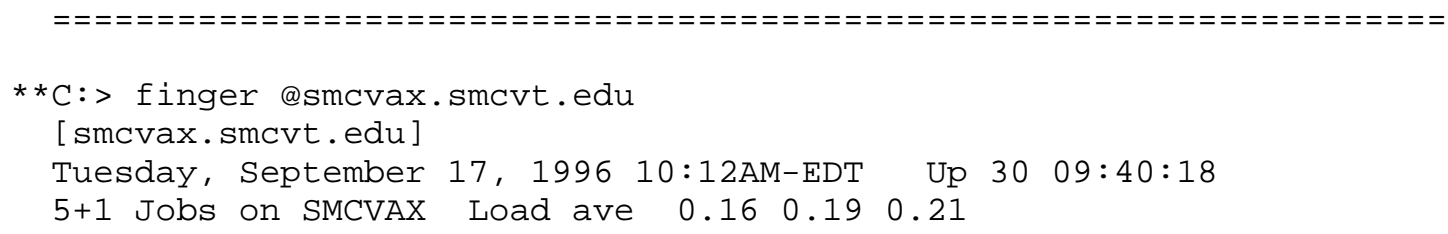




\begin{tabular}{|c|c|c|c|c|}
\hline User & Personal Name & Subsys & Terminal & Console Location \\
\hline GOODWIN & Dave Goodwin & LYNX & 6.NTY2 & waldo.smcvt.edu \\
\hline JAT & John Tronoan & TELNET & 1. TXA5 & \\
\hline HELPDESK & System Manager & $\mathrm{EDT}$ & $2: 08 . N T Y 4$ & [199.93.35.182] \\
\hline SMITH & Lorraine Smith & PINE & .NTY3 & {$[199.93 .34 .139]$} \\
\hline SYSTEM & System Manager & $\begin{array}{l}\text { MAIL } \\
\text { *DCL* }\end{array}$ & $\begin{array}{c}23 . \text { OPAO } \\
\text { SMCVX1 SOPA0 }\end{array}$ & $\begin{array}{l}\text { The VAX Console } \\
\text { The VAX Console }\end{array}$ \\
\hline
\end{tabular}

\subsection{Traceroute}

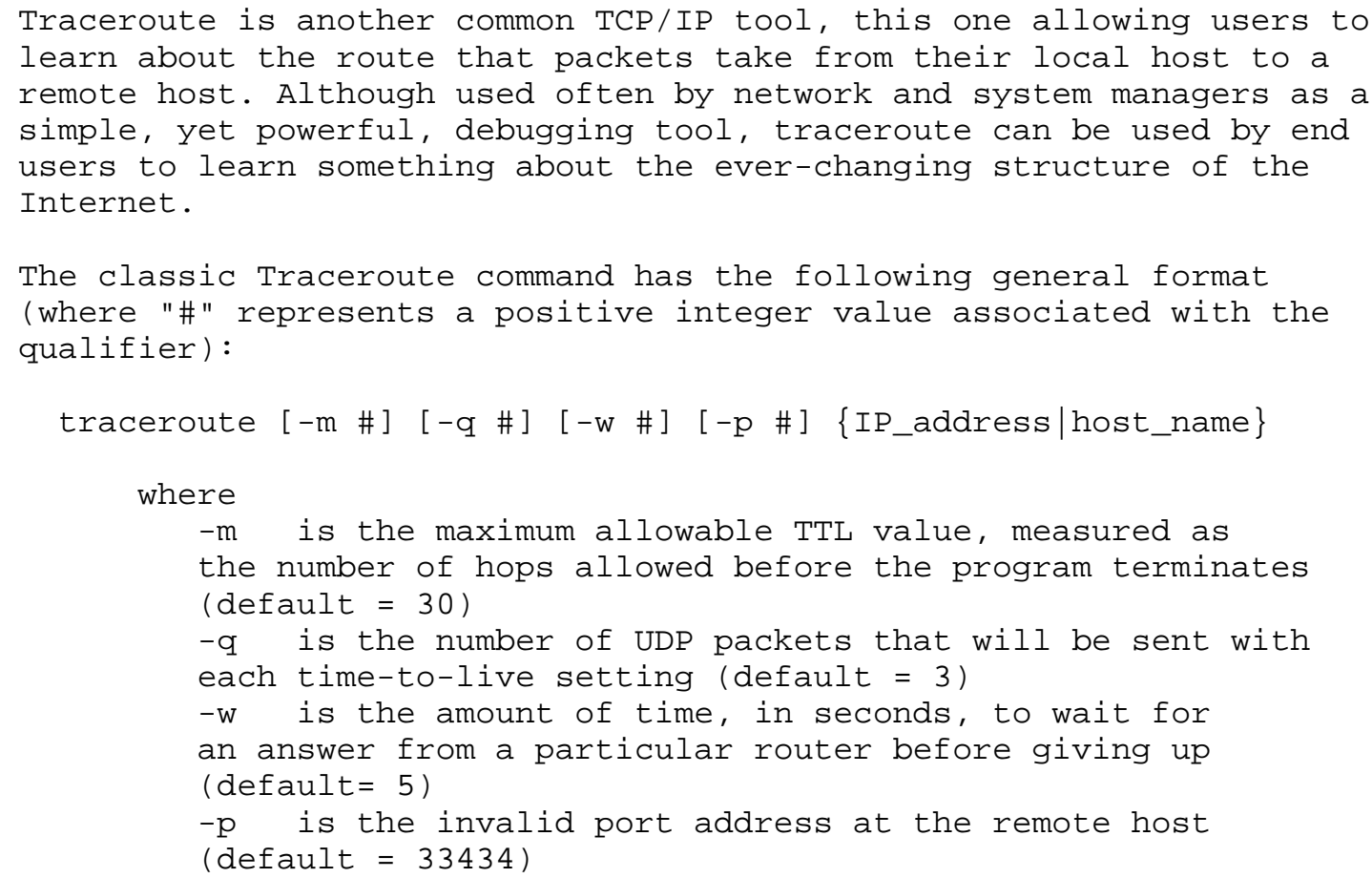

The Traceroute example below shows the route between a host at St. Michael's College (domain smcvt.edu) and a host at Hill Associates (www.hill.com), both located in Colchester, VT but served by different Internet service providers (ISP). 
1 St. Michael's College is connected to the Internet via BBN Planet; since the mid-1980s, BBN operated the NSF's regional ISP, called the New England Academic and Research Network (NEARNET), which was renamed in 1994. The first hop, then, goes to St. Mike's BBN Planet gateway router (smc.bbnplanet.net). The next hop goes to another BBN Planet router (denoted here only by IP address since a name was not assigned to the device), until the packet reaches the BBN Planet T3 backbone.

2 The packet takes two hops through routers at BBN Planet's Cambridge (MA) facility and is then forwarded to BBN Planet in New York City, where the packet takes four more hops. The packet is then forwarded to BBN Planet in College Park (MD).

3 The packet is sent to BBN Planet's router at MAE-East, MFS Datanet's Network Access Point (NAP) in Washington, D.C. MAE stands for Metropolitan Area Exchange, and is a Fiber Distributed Data Interface (FDDI) ring interconnecting routers from subscribing ISPS. The packet is then forwarded to NETCOM, Hill Associates' ISP.

4 The packet now travels through NETCOM's T3 backbone, following links from Washington, D.C. to Chicago to Santa Clara (CA), to San Jose (CA).

5 The packet is now sent to Hill Associates router (again, a system designated only by an IP address since the NETCOM side of the router was not named) and then passed to the target system. Note that the host's real name is not www.hill.com, but syrup.hill.com.

TECHNICAL NOTE: The original version of Traceroute works by sending a sequence of User Datagram Protocol (UDP) datagrams to an invalid port address at the remote host. Using the default settings, three datagrams are sent, each with a Time-To-Live (TTL) field value set to one. The TTL value of 1 causes the datagram to "timeout" as soon as it hits the first router in the path; this router will then respond with an ICMP Time Exceeded Message (TEM) indicating that the datagram has expired. Another three UDP messages are now sent, each with the TTL value set to 2, which causes the second router to return ICMP 
TEMs. This process continues until the packets actually reach the other destination. Since these datagrams are trying to access an invalid port at the destination host, ICMP Destination Unreachable Messages are returned indicating an unreachable port; this event signals the Traceroute program that it is finished! The Traceroute program displays the round-trip delay associated with each of the attempts. (Note that some current implementations of Traceroute use the Record-Route option in IP rather than the method described above. )

As an aside, Traceroute did not begin life as a general-purpose utility, but as a quick-and-dirty debugging aid used to find a routing problem. The code (complete with comments!) is available by anonymous FTP in the file traceroute.tar.z from the host ftp.ee.lbl.gov. (See Section 4.2 for a discussion of anonymous FTP.)

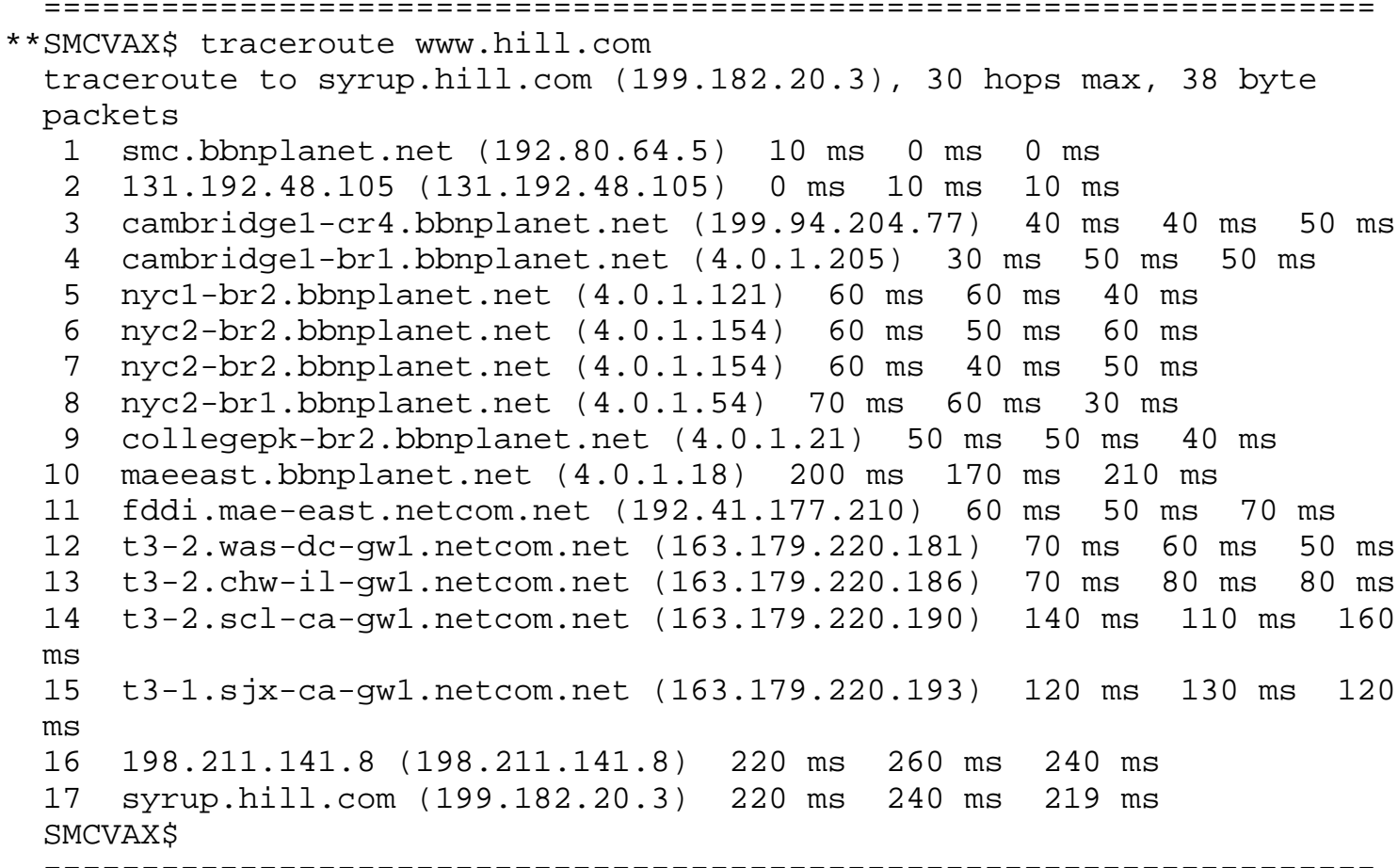




\section{The Two Fundamental Tools}

The two most basic tools for Internet applications are TELNET and the File Transfer Protocol (FTP). TELNET allows a user to login to a remote host over a TCP/IP network, while FTP, as the name implies, allows a user to move files between two TCP/IP hosts. These two utilities date back to the very early days of the ARPANET.

\subsection{TELNET}

TELNET [27] is TCP/IP's virtual terminal protocol. Using TELNET, a user connected to one host can login to another host, appearing like a directly-attached terminal at the remote system; this is TCP/IP's definition of a virtual terminal. The general form of the TELNET command is:

telnet [IP_address|host_name] [port]

As shown, a TELNET connection is initiated when the user enters the telnet command and supplies either a host_name or IP_address; if neither are given, TELNET will ask for one once the application begins.

In the example below, a user of a PC uses TELNET to attach to the remote host smcvax.smcvt.edu. Once logged in via TELNET, the user can do anything on the remote host that would be possible if connected via a directly-attached terminal or via modem. The commands that are subsequently used are those available on the remote system to which the user is attached. In the sample dialogue below, the user attached to SMCVAX will use basic VAX/VMS commands:

- The dir command lists the files having a "COM" file extension. - The mail command enters the VMS MAIL subsystem; the dir command here lists waiting mail.

o Ping checks the status of another host.

When finished, the logout command logs the user off the remote host; TELNET automatically closes the connection to the remote host and returns control to the local system.

It is important to note that TELNET is a very powerful tool, one that may provide users with access to many Internet utilities and services that might not be otherwise available. Many of these features are accessed by specifying a port number with the TELNET command, in addition to a host's address, and knowledge of port numbers provides another mechanism for users to access information with TELNET. 
This guide discusses several TCP/IP and Internet utilities that require local client software, such as Finger, Whois, Archie, and Gopher. But what if your software does not include a needed client? In some cases, TELNET may be used to access a remote client and provide the same functionality.

This is done by specifying a port number with the TELNET command. Just as TCP/IP hosts have a unique IP address, applications on the host are associated with an address, called a port. Finger (see Section 3.3 above), for example, is associated with the well-known port number 79 . In the absence of a Finger client, TELNETing to port 79 at a remote host may provide the same information. You can finger another host with TELNET by using a command like:

$$
\text { telnet host_name } 79
$$

Other well-known TCP port numbers include 25 (Simple Mail Transfer Protocol), 43 (whois), 80 (Hypertext Transfer Protocol), and 119 (Network News Transfer Protocol).

Some services are available on the Internet using TELNET and special port numbers. A geographical information database, for example, may be accessed by TELNETing to port 3000 at host martini.eecs.umich.edu and current weather information is available at port 3000 at host downwind.sprl.umich.edu.

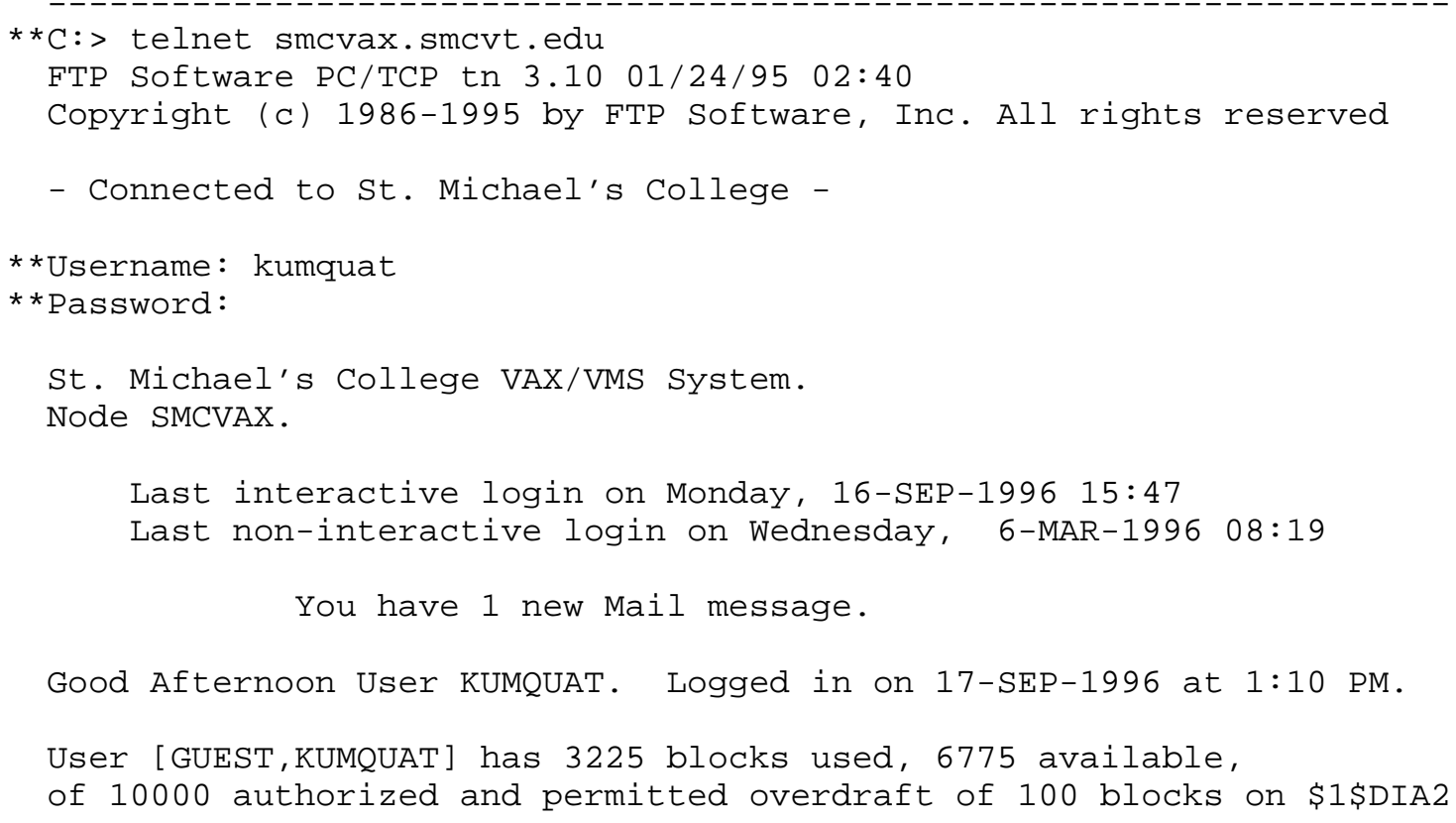




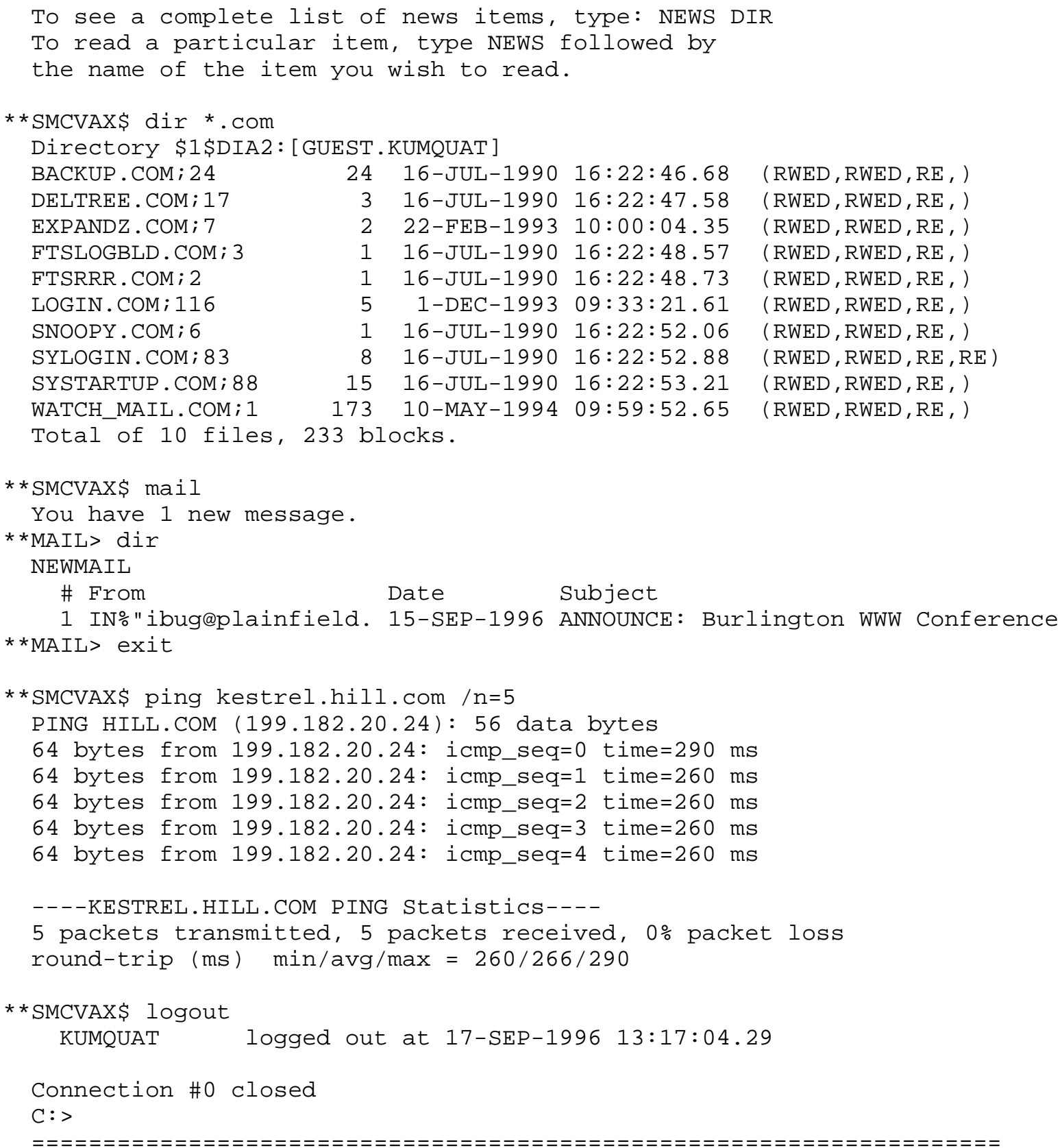




\subsection{FTP}

FTP [26] is one of the most useful and powerful TCP/IP utilities for the general user. FTP allows users to upload and download files between local and remote hosts. Anonymous FTP, in particular, is commonly available at file archive sites to allow users to access files without having to pre-establish an account at the remote host. TELNET might, in fact, be used for this purpose but TELNET gives the user complete access to the remote system; FTP limits the user to file transfer activities.

The general form of the FTP command is:

$$
\text { ftp [IP_address|host_name] }
$$

An FTP session can be initiated in several ways. In the example shown below, an FTP control connection is initiated to a host (the Defense Data Network's Network Information Center) by supplying a host name with the FTP command; optionally, the host's IP address in dotted decimal (numeric) form could be used. If neither host name nor IP address are supplied in the command line, a connection to a host can be initiated by typing open host_name or open IP_address once the FTP application has been started.

The remote host will ask for a username and password. If a bona fide registered user of this host supplies a valid username and password, then the user will have access to any files and directories to which this username has privilege. For anonymous FTP access, the username anonymous is used. Historically, the password for the anonymous user (not shown in actual use) has been guest, although most systems today ask for the user's Internet e-mail address (and several sites attempt to verify that packets are coming from that address before allowing the user to login).

The "help ?" command may be used to obtain a list of FTP commands and help topics available with your software; although not always shown, nearly all TCP/IP applications have a help command. An example of the help for FTP's type command is shown in the sample dialogue. This command is very important one, by the way; if transferring a binary or executable file, be sure to set the type to image (or binary on some systems).

The dir command provides a directory listing of the files in the current directory at the remote host; the UNIX ls command may also usually be used. Note that an FTP data transfer connection is established for the transfer of the directory information to the local host. The output from the dir command will show a file listing that is consistent with the native operating system of the remote 
host. Although the TCP/IP suite is often associated with UNIX, it can (and does) run with nearly all common operating systems. The directory information shown in the sample dialogue happens to be in UNIX format and includes the following information:

o File attributes. The first character identifies the type of file entry as a directory (d), link or symbolic name (1), or individual file (-). The next nine characters are the file access permissions list; the first three characters are for the owner, the next three for the owner's group, and the last three for all other users. Three access privileges may be assigned to each file for each of these roups: read ( $r)$, write (w), and execute (x). - Number of entries, or hard links, in this structure. This value will be a "1" if the entry refers to a file or link, or will be the number of files in the listed directory.

o File owner

o File owner's group.

- File size, in bytes.

- Date and time of last modification. If the date is followed by a timestamp, then the date is from the current year.

o File name.

After the directory information has been transferred, FTP closes the data transfer connection.

The command $\mathrm{cd}$ is used to change to another working directory, in this case the rfc directory (note that file and directory names may be case-sensitive). As in DOS, "cd.." will change to the parent of the current directory. The CWD command successful is the only indication that the user's cd command was correctly executed; the show-directory (may be truncated to fewer characters, as shown) command, if available, may be used to see which working directory you are in.

Another dir command is used to find all files with the name rfc173*.txt; note the use of the * wildcard character. We can now copy (download) the file of choice (RFC 1739 is the previous version of this primer) by using the get (or receive) command, which has the following general format:

get remote_file_name local_file_name

FTP opens another data transfer connection for this file transfer purpose; note that the effective data transfer rate is $93.664 \mathrm{kbps}$.

FTP's put (or send) command allows uploading from the local host to the remote. Put is often not available when using anonymous FTP. 
Finally, we terminate the FTP connection by using the close command. The user can initiate another FTP connection using the open command or can leave FTP by issuing a quit command. Quit can also be used to close a connection and terminate a session.

TECHNICAL NOTE: It is important to note that different FTP packages have different commands available and even those with similar names may act differently. In the example shown here (using MultiNet for VMS), the show command will display the current working directory; in FTP Software's OnNet, show will display a file from the remote host at the local host. Some packages have nothing equivalent to either of these commands.

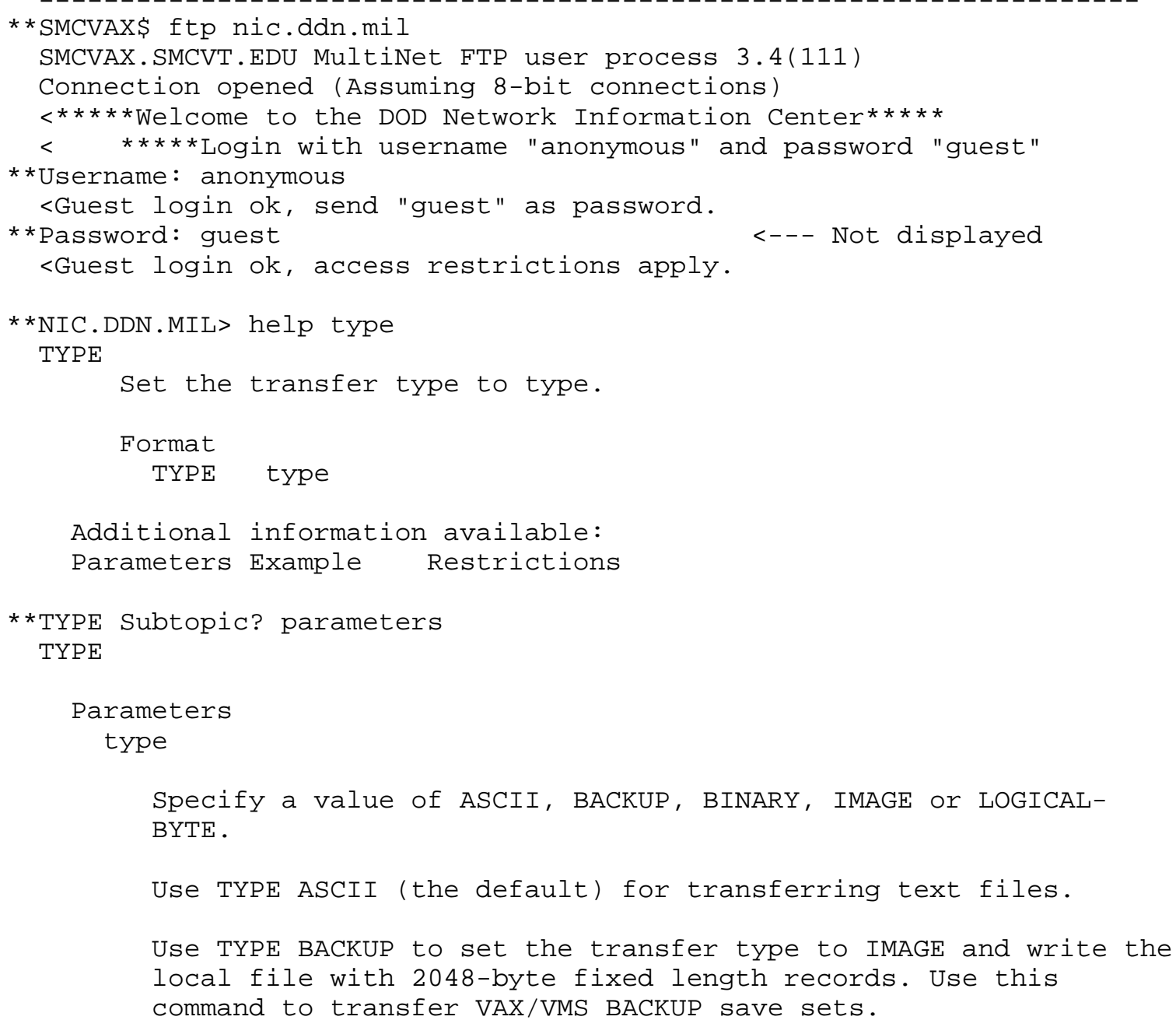




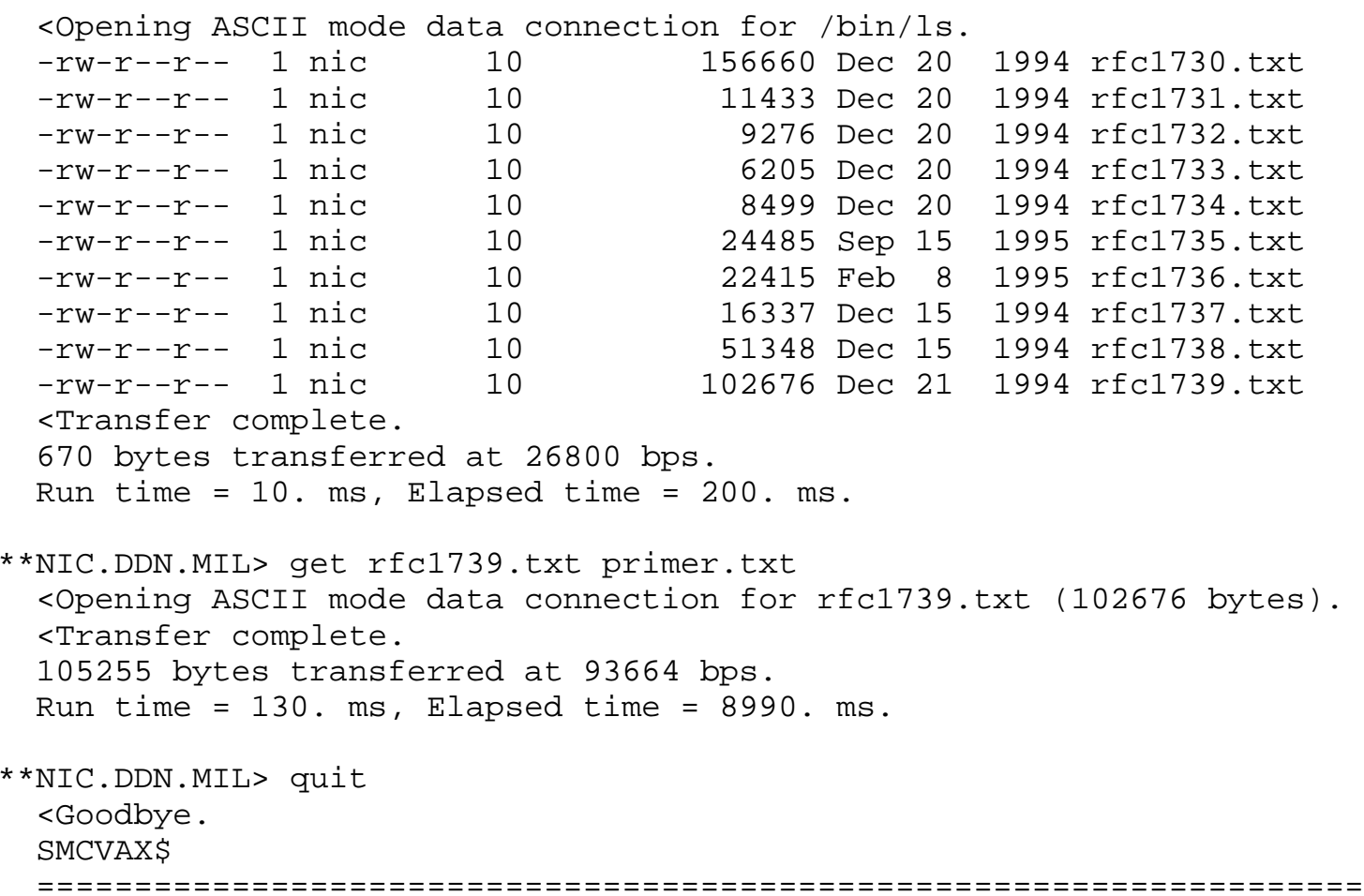

5. User Database Lookup Tools

Finding other users on the Internet is an art, not a science. Although there is a distributed database listing all of the 16+ million hosts on the Internet, no similar database yet exists for the tens of millions of users. While many commercial ISPs provide directories of the users of their network, these databases are not yet linked. The paragraphs below will discuss some of the tools available for finding users on the Internet.

\subsection{WHOIS / NICNAME}

WHOIS and NICNAME are TCP/IP applications that search databases to find the name of network and system administrators, RFC authors, system and network points-of-contact, and other individuals who are registered in appropriate databases. The original NICNAME/WHOIS protocol is described in RFC 954 [10].

WHOIS may be accessed by TELNETing to an appropriate WHOIS server and logging in as whois (no password is required); the most common Internet name server is located at the Internet Network Information Center (InterNIC) at rs.internic.net. This specific database only 
contains INTERNET domains, IP network numbers, and domain points of contact; policies governing the InterNIC database are described in RFC 1400 [31]. The MILNET database resides at nic.ddn.mil and PSI'S White Pages pilot service is located at psi.com.

Many software packages contain a WHOIS/NICNAME client that automatically establishes the TELNET connection to a default name server database, although users can usually specify any name server database that they want.

The accompanying dialogues shows several types of WHOIS/NICNAME information queries. In the session below, we request information about an individual (Denis stratford) by using WHOIS locally, a specific domain (hill.com) by using NICNAME locally, and a network address (199.182.20.0) and high-level domain (com) using TELNET to a WHOIS server.

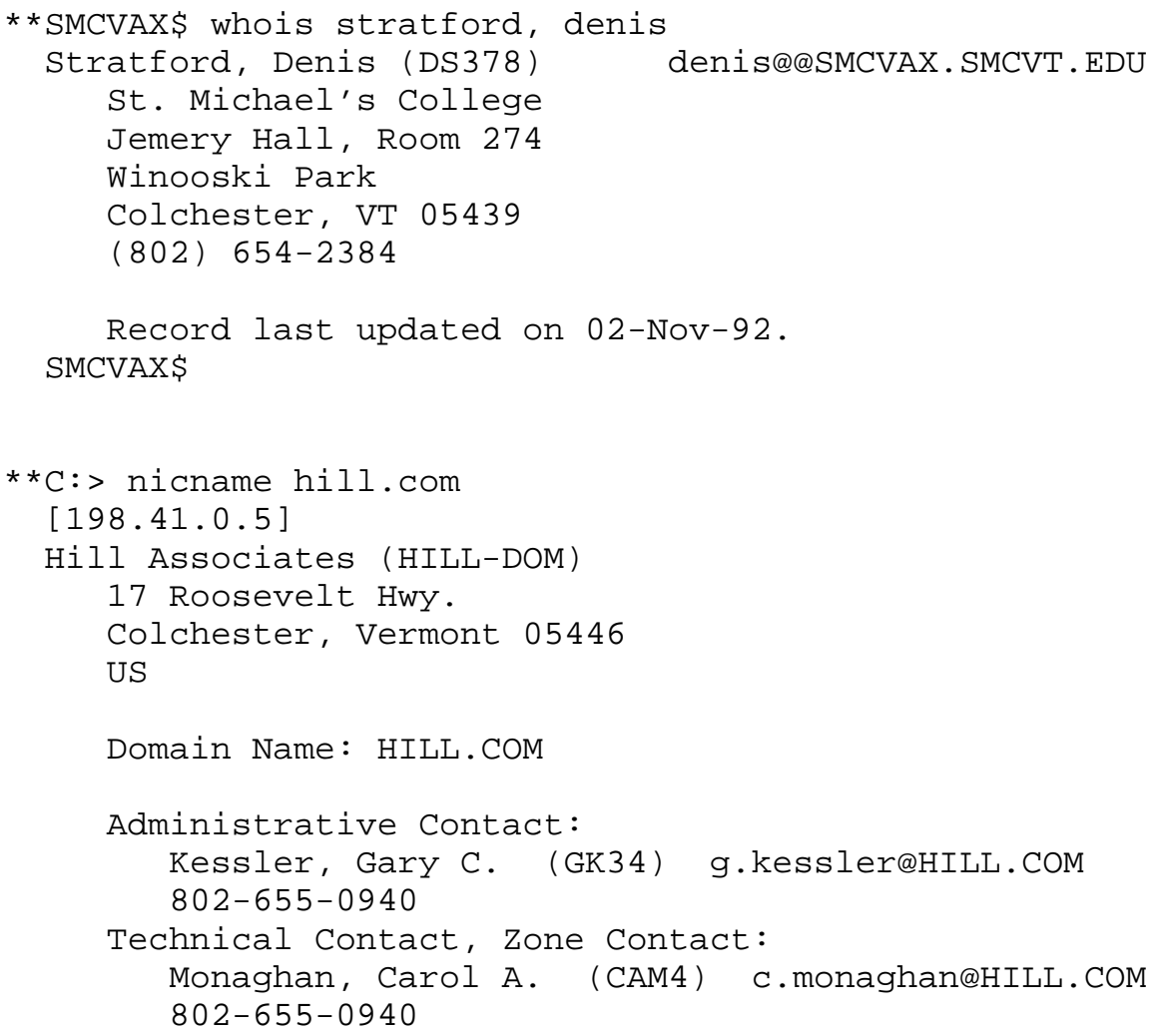




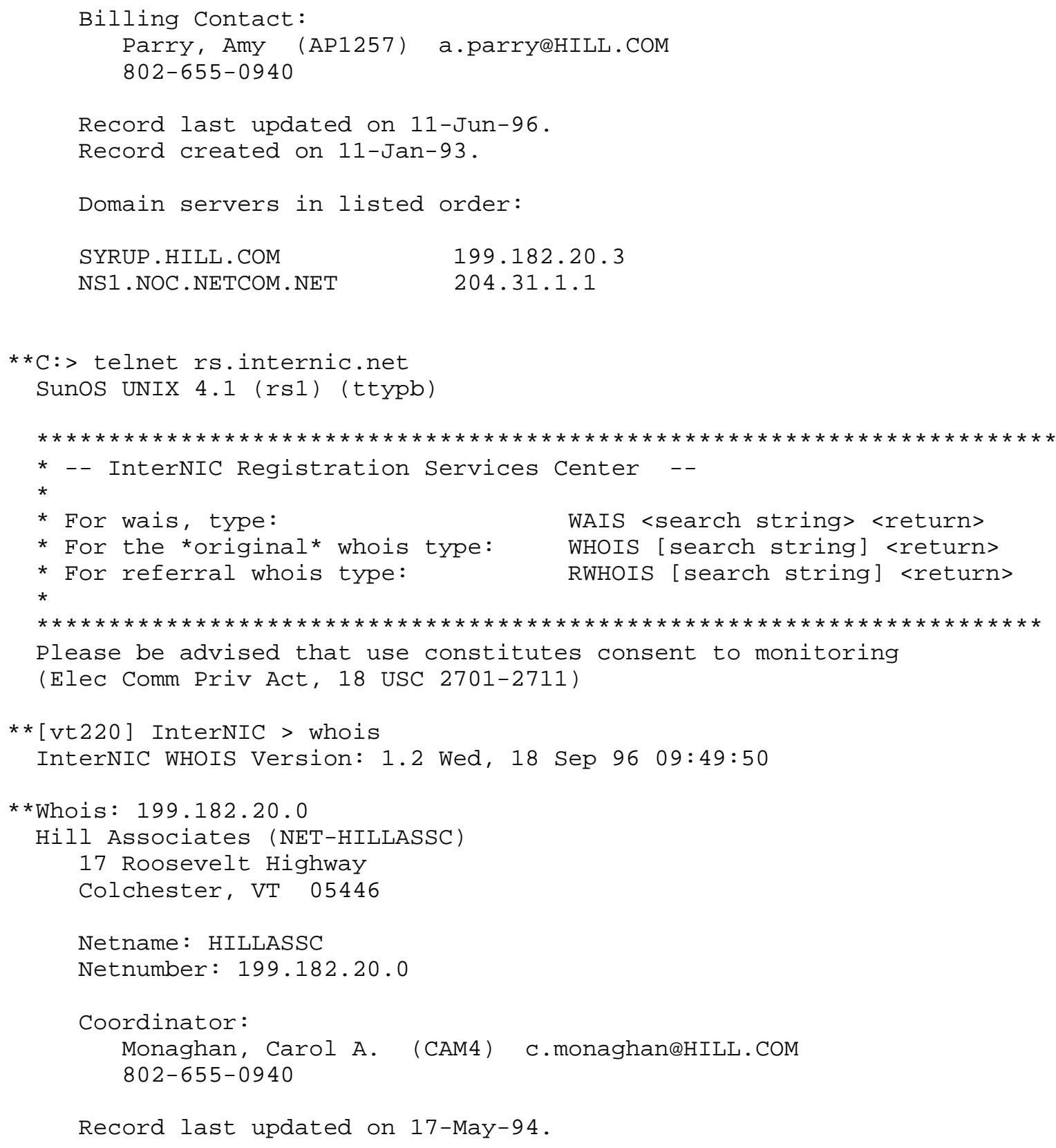




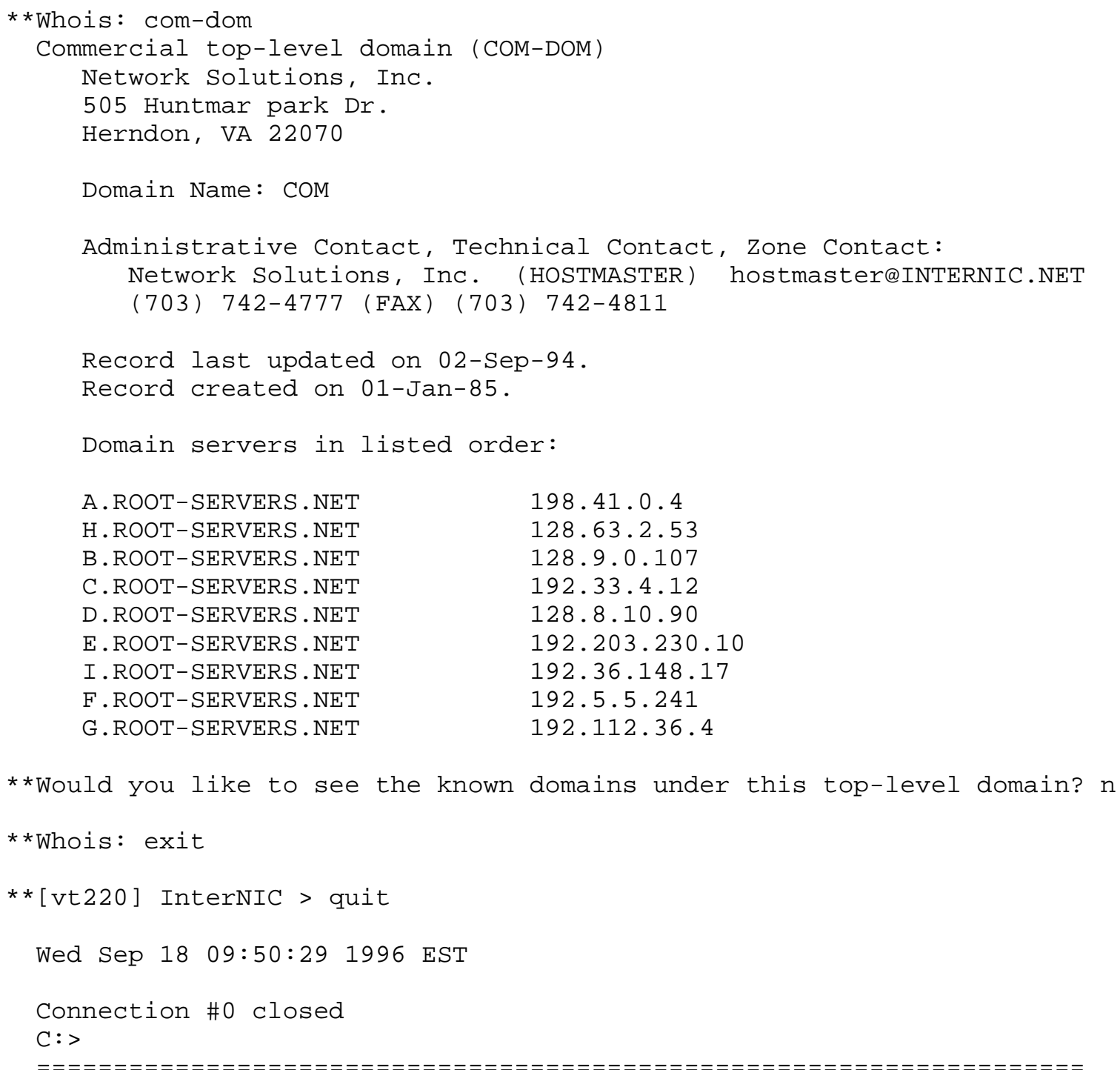




\subsection{KNOWBOT}

KNOWBOT is an automated username database search tool that is related to WHOIS. The Knowbot Information Service (KIS), operated by the Corporation for National Research Initiatives (CNRI) in Reston, Virginia, provides a simple WHOIS-like interface that allows users to query several Internet user databases (White Pages services) all at one time. A single KIS query will automatically search the InterNIC, MILNET, MCImail, and PSI White Pages Pilot Project; other databases may also be included.

KNOWBOT may be accessed by TELNETing to host info.cnri.reston.va.us. The help command will supply sufficient information to get started. The sample dialogue below shows use of the query command to locate a user named "Steven Shepard"; this command automatically starts a search through the default set of Internet databases.

${ }^{*} \mathrm{C}:>$ telnet info.cnri.reston.va.us

Knowbot Information Service

KIS Client (V2.0). Copyright CNRI 1990. All Rights Reserved.

KIS searches various Internet directory services

to find someone's street address, email address and phone number.

Type 'man' at the prompt for a complete reference with examples.

Type 'help' for a quick reference to commands.

Type 'news' for information about recent changes.

Please enter your email address in our guest book...

** (Your email address?) > s.shepard@hill.com

**> query shepard, steven

Trying whois at ds.internic.net...

The ds.internic.net whois server is being queried:

Nothing returned.

The rs.internic.net whois server is being queried:

Shepard, Steven (SS2192) 708-810-5215

Shepard, Steven (SS1302) axisteven@AOL.COM (954) 974-4569 
The nic.ddn.mil whois server is being queried:

Shepard, Steven (SS2192)

R.R. Donnelley \& Sons

750 Warrenville Road

Lisle, IL 60532

Trying mcimail at cnri.reston.va.us...

Trying ripe at whois.ripe.net...

Trying whois at whois.lac.net...

No match found for . SHEPARD, STEVEN

$\star *>$ quit

KIS exiting

Connection \#0 closed

$\mathrm{C}:>$

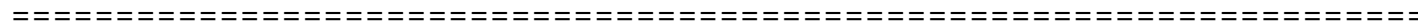

6. Information Servers

File transfer, remote login, and electronic mail remained the primary applications of the ARPANET/Internet until the early 1990s. But as the Internet user population shifted from hard-core computer researchers and academics to more casual users, easier-to-use tools were needed for the Net to become accepted as a useful resource. That means making things easier to find. This section will discuss some of the early tools that made it easier to locate and access information on the Internet.

\subsection{Archie}

Archie, developed in 1992 at the Computer Science Department at McGill University in Montreal, allows users to find software, data, and other information files that reside at anonymous FTP archive sites; the name of the program, reportedly, is derived from the word "archive" and not from the comic book character. Archie tracks the contents of several thousand anonymous FTP sites containing millions of files. The archie server automatically updates the information from each registered site about once a month, providing relatively up-to-date information without unduly stressing the network. Archie, however, is not as popular as it once was and many sites have not updated their information; as the examples below show, many of the catalog listings are several years old. 
Before using archie, you must identify a server address. The sites below all support archie; most (but not all) archie sites support the servers command which lists all known archie servers. Due to the popularity of archie at some sites and its high processing demands, many sites limit access to non-peak hours and/or limit the number of simultaneous archie users. Available archie sites include:

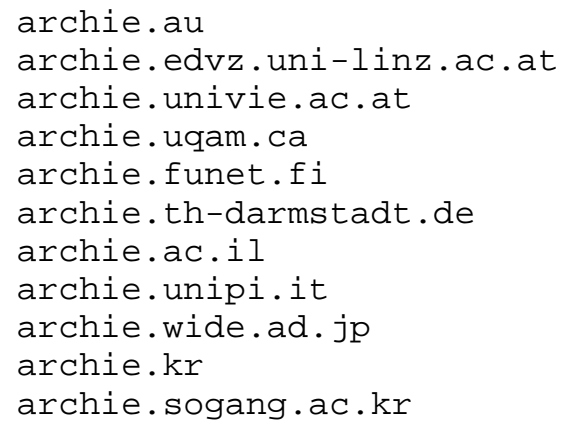

All archie sites can be accessed using archie client software. Some archie servers may be accessed using TELNET; when TELNETing to an archie site, login as archie (you must use lower case) and hit $<$ ENTER> if a password is requested.

Once connected, the help command assists users in obtaining more information about using archie. Two more useful archie commands are prog, used to search for files in the database, and whatis, which searches for keywords in the program descriptions.

In the accompanying dialogue, the set maxhits command is used to limit the number of responses to any following prog commands; if this is not done, the user may get an enormous amount of information. In this example, the user issues a request to find entries related to "dilbert"; armed with this information, a user can use anonymous FTP to examine these directories and files.

The next request is for files with "tcp/ip" as a keyword descriptor. These responses can be used for subsequent prog commands.

Exit archie using the exit command. At this point, TELNET closes the connection and control returns to the local host. 
Additional information about archie can be obtained by sending e-mail to Bunyip Information Systems (archie-info@bunyip.com). Client software is not required to use archie, but can make life a little easier; some such software can be downloaded using anonymous FTP from the /pub/archie/clients/ directory at ftp.sura.net (note that the newest program in this directory is dated June 1994). Most shareware and commercial archie clients hide the complexity described in this section; users usually connect to a pre-configured archie server merely by typing an archie command line.

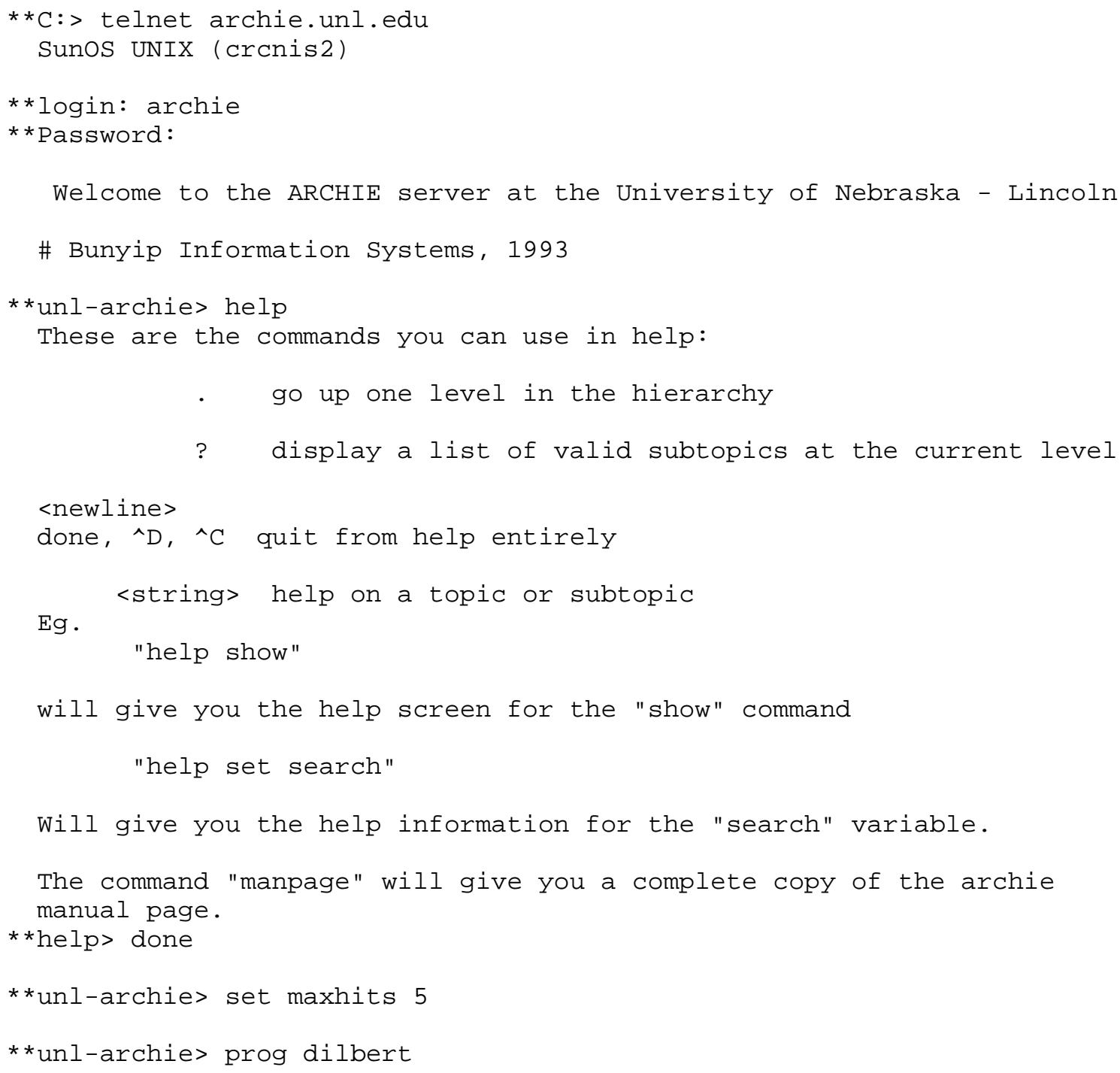




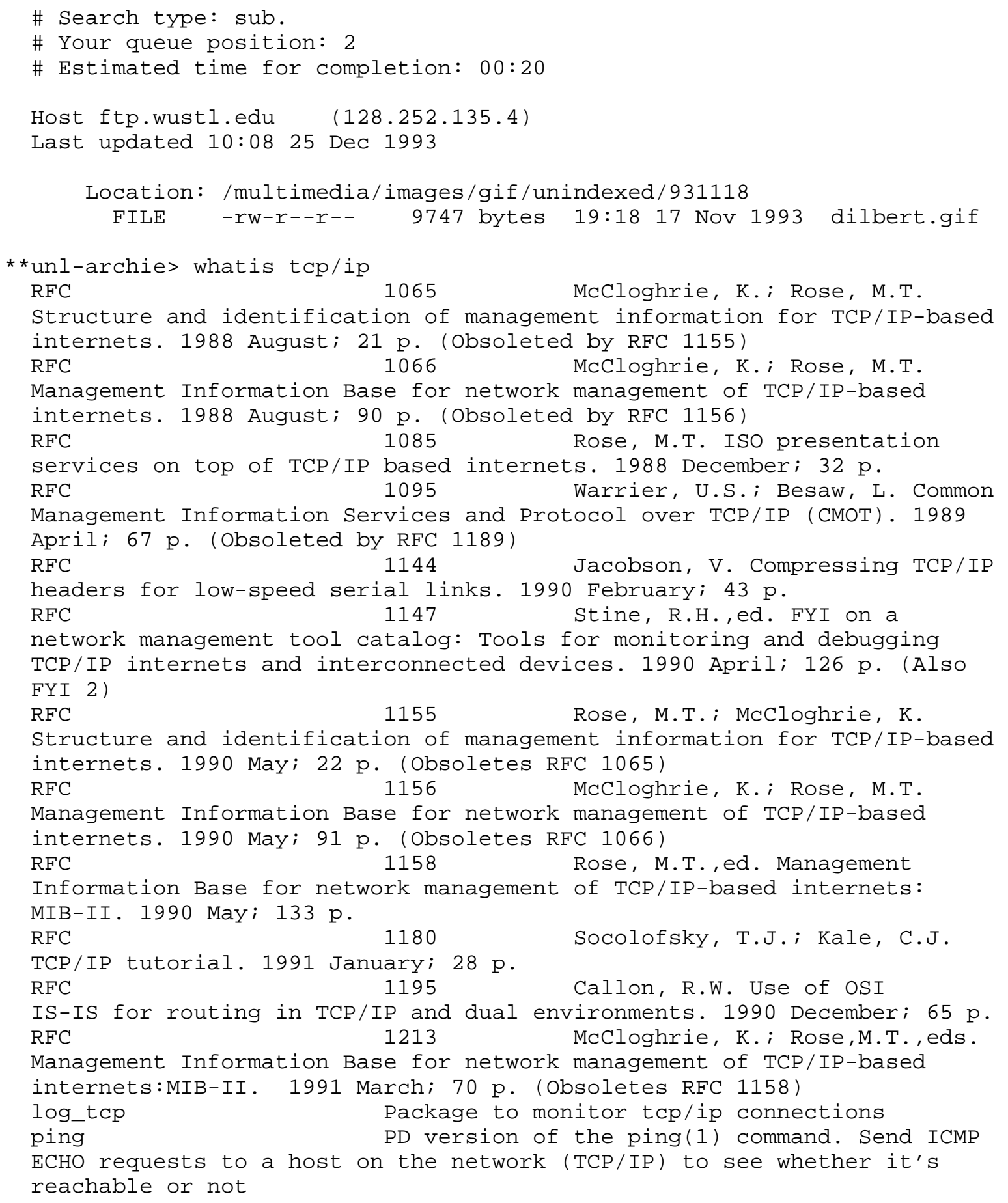




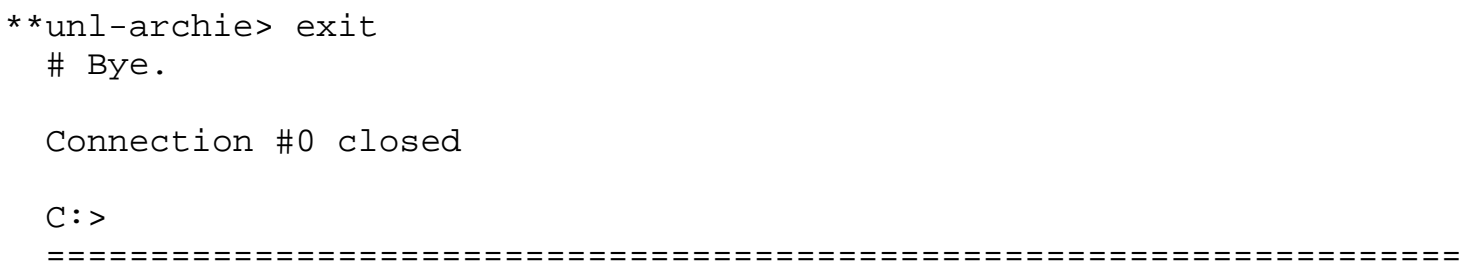

\subsection{Gopher}

The Internet Gopher protocol was developed at the University of Minnesota's Microcomputer Center in 1991, as a distributed information search and retrieval tool for the Internet. Gopher is described in RFC 1436 [1]; the name derives from the University's mascot.

Gopher provides a tool so that publicly available information at a host can be organized in a hierarchical fashion using simple text descriptions, allowing files to be perused using a simple menu system. Gopher also allows a user to view a file on demand without requiring additional file transfer protocols. In addition, Gopher introduced the capability of linking sites on the Internet, so that each Gopher site can be used as a stepping stone to access other sites and reducing the amount of duplicate information and effort on the network.

Any Gopher site can be accessed using Gopher client software (or a WWW browser). In many cases, users can access Gopher by TELNETing to a valid Gopher location; if the site provides a remote Gopher client, the user will see a text-based, menu interface. The number of Gopher sites grew rapidly between 1991 and 1994, although growth tapered due to the introduction of the Web; in any case, most Gopher sites have a menu item that will allow you to identify other Gopher sites. If using TELNET, login with the username gopher (this must be in lowercase); no password is required.

In the sample dialogue below, the user attaches to the Gopher server at the Internet Network Information Center (InterNIC) by TELNETing to ds.internic.net. With the menu interface shown here, the user merely follows the prompts. Initially, the main menu will appear. Selecting item 3 causes Gopher to seize and display the "InterNIC Registration Services (NSI)" menu; move to the desired menu item by typing the item number or by moving the pointer $(-->)$ down to the desired entry using the DOWN-ARROW key on the keyboard, and then hitting ENTER. To quit the program at any time, press q (quit); ? and u will provide help or go back up to the previous menu, respectively. Users may also search for strings within files using the / command or download the file being interrogated using the D command. 
Menu item 1 within the first submenu (selected in the dialogue shown here) is titled "InterNIC Registration Archives." As its submenu implies, this is a place to obtain files containing the InterNIC's domain registration policies, domain data, registration forms, and other information related to registering names and domains on the Internet.

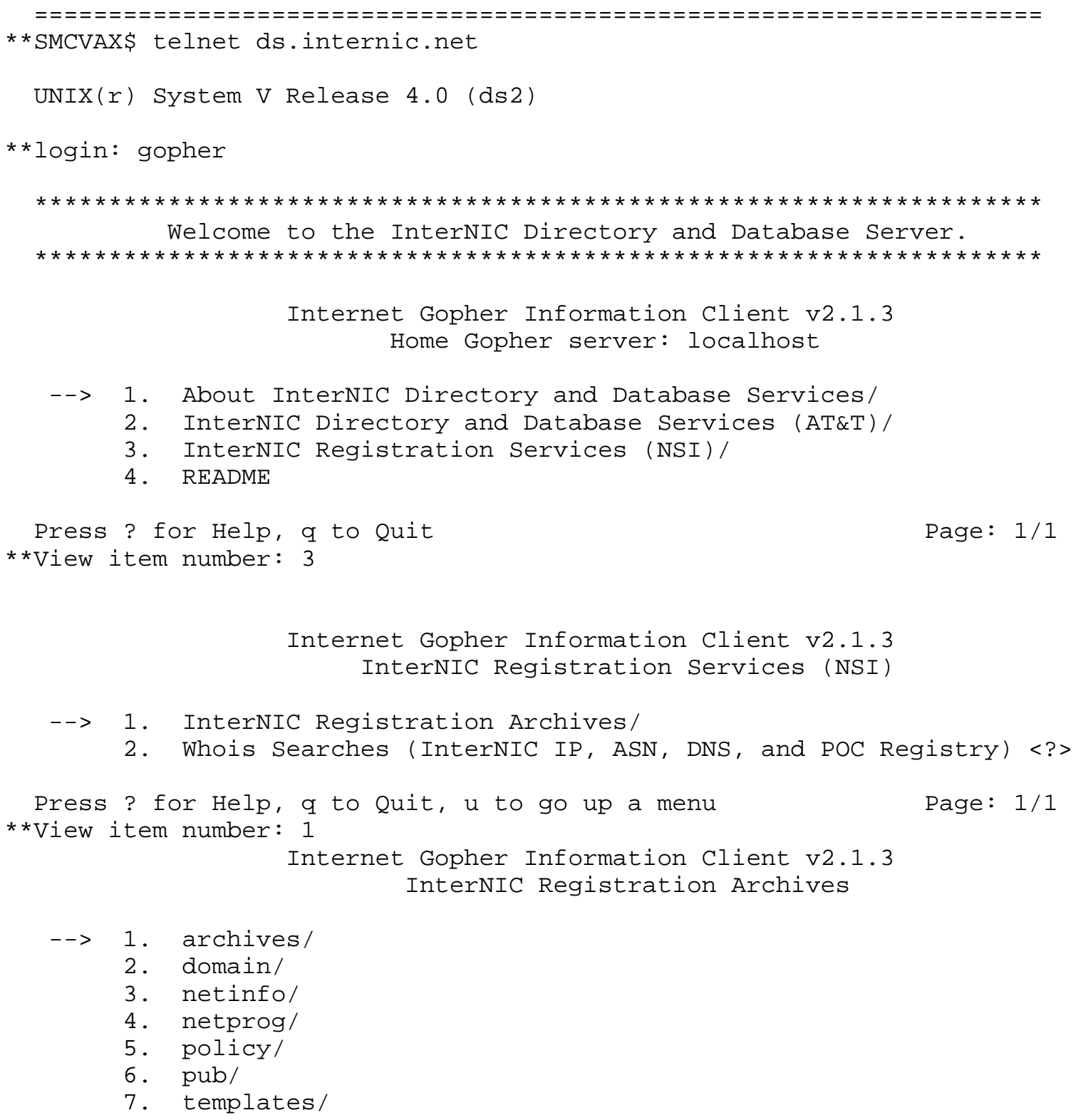


Press ? for Help, q to Quit, u to go up a menu

Page: $1 / 1$ $\star \star \mathrm{q}$

$\star \star$ Really quit (y/n) ? y

Connection closed by Foreign Host

SMCVAXS

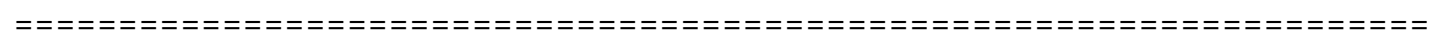

6.3. VERONICA, JUGHEAD, and WAIS

The problem with being blessed with so much information from FTP, archie, Gopher, and other sources is exactly that -- too much information. To make it easier for users to locate the system on which their desired information resides, a number of other tools have been created.

VERONICA (Very Easy Rodent-Oriented Net-wide Index to Computerized Archives) was developed at the University of Nevada at Reno as an archie- like adjunct to Gopher. As the number of Gopher sites quickly grew after its introduction, it became increasingly harder to find information in gopherspace since Gopher was designed to search a single database at a time. VERONICA maintains an index of titles of Gopher items and performs a keyword search on all of the Gopher sites that it has knowledge of and access to, obviating the need for the user to perform a menu-by-menu, site-by-site search for information. When a user selects an item from the menu of a VERONICA search, "sessions" are automatically established with the appropriate Gopher servers, and a list of data items is returned to the originating Gopher client in the form of a Gopher menu so that the user can access the files. VERONICA is available as an option on many Gopher servers.

Another Gopher-adjunct is JUGHEAD (Jonzy's Universal Gopher Hierarchy Excavation And Display). JUGHEAD supports key word searches and the use of logical operators (AND, OR, and NOT). The result of a JUGHEAD search is a display of all menu items which match the search string which are located in the University of Manchester and UMIST Information Server, working from a static database that is re-created every day. JUGHEAD is available from many Gopher sites, although VERONICA may be a better tool for global searches.

The Wide Area Information Server (WAIS, pronounced "ways") was initiated jointly by Apple Computer, Dow Jones, KMPG Peat Marwick, and Thinking Machines Corp. It is a set of free-ware, share-ware, and commercial software products for a wide variety of hardware/software platforms, which work together to help users find information on the Internet. WAIS provides a single interface through which a user can 
access many different information databases. The user interface allows a query to be formulated in English and the WAIS server will automatically choose the appropriate databases to search. Further information about WAIS can be obtained by reading the WAIS FAQ, from host rtfm.mit.edu in file/pub/usenet/news.answers/wais-faq.

7. The World Wide Web

The World Wide Web (WWW) is thought (erroneously) by many to be the same thing as the Internet. But the confusion, in many ways, is justified; by early 1996, the WWW accounted for over $40 \%$ of all of the traffic on the Internet. In addition, the number of hosts on the Internet named www has grown from several hundred in mid-1994 to 17,000 in mid-1995 to 212,000 in mid-1996 to over 410,000 by early 1997. The Web has made information on the Internet accessible to users of all ages and computer skill levels. It has provided a mechanism so that nearly anyone can become a content provider. According to some, growth in the number of WWW users is unparalleled by any other event in human history.

The WWW was developed in the early 1990s at the CERN Institute for Particle Physics in Geneva, Switzerland. The Web was designed to combine aspects of information retrieval with multimedia communications, unlike archie and Gopher, which were primarily used for the indexing of text-based files. The Web allows users to access information in many different types of formats, including text, sound, image, animation, and video. WWW treats all searchable Internet files as hypertext documents. Hypertext is a term which merely refers to text that contains pointers to other text, allowing a user reading one document to jump to another document for more information on a given topic, and then return to the same location in the original document. WWW hypermedia documents are able to employ images, sound, graphics, video, and animation in addition to text.

To access WWW servers, users must run client software called a browser. The browser and server use the Hypertext Transfer Protocol (HTTP) [3]. WWW documents are written in the Hypertext Markup Language (HTML) [2, 20], a simple text-based formatting language that is hardware and software platform-independent. Users point the browser at some location using a shorthand format called a Uniform Resource Locator (URL), which allows a WWW servers to obtain files from any location on the public Internet using a variety of protocols, including HTTP, FTP, Gopher, and TELNET.

Mosaic, developed in 1994 at the National Center for supercomputer Applications (NCSA) at the University of Illinois at UrbanaChampaign, was the first widely-used browser. Because it was available at no cost over the Internet via anonymous FTP, and had a 
version for Windows, Mac, and UNIX systems, Mosaic was probably the single reason that the Web attracted so many users so quickly. The most commonly used browsers today include the Netscape Navigator (http://www.netscape.com), Microsoft's Internet Explorer (http://www.microsoft.com), and NCSA Mosaic (http://www.ncsa.uiuc.edu/SDG/Software/Mosaic/).

The WWW is ideally suited to a windows environment, or other pointand-click graphical user interface. Nevertheless, several text-based Web browsers do exist, although their usefulness is limited if trying to obtain graphical images, or audio or video clips. One text-based Web browser is Lynx, and an example of its use is shown below. Items in square brackets in the sample dialogue are Lynx's way of indicating an image or other display that cannot be shown on an ASCII terminal.

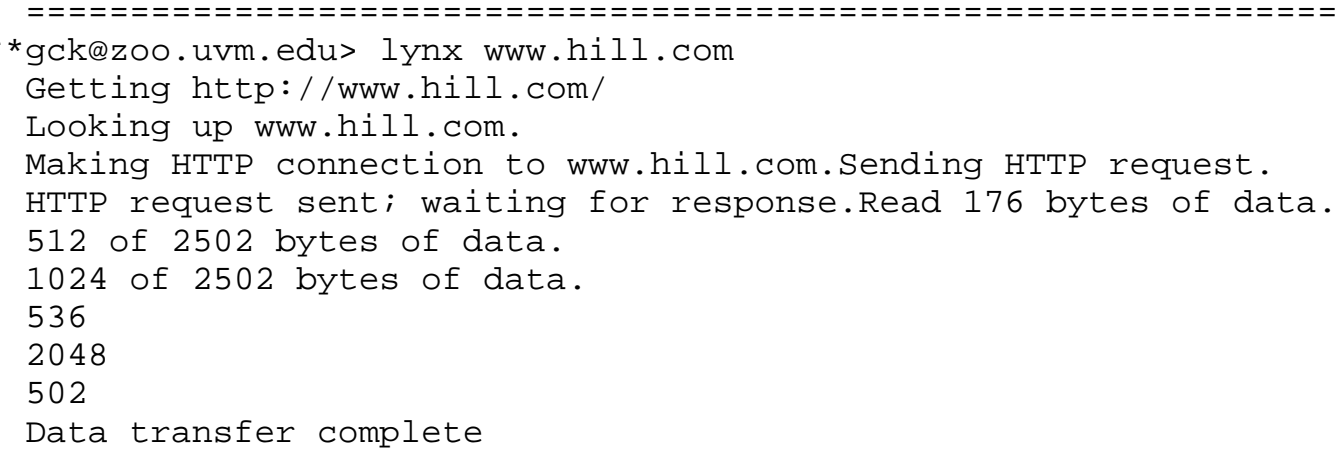

Hill Associates

[INLINE] Hill Associates, Inc. Leaders in Telecommunications Training and Education Worldwide

Hill Associates is an international provider of voice and data telecommunications training and education. We cover the full breadth of the field, including telephony, computer networks, ISDN, X.25 and fast packet technologies (frame relay, SMDS, ATM), wireless, TCP/IP and the Internet, LANs and LAN interconnection, legacy networks, multimedia and virtual reality, broadband services, regulation, service strategies, and network security.

Hill Associates' products and services include instructor-led, computer-based (CBT), and hands-on workshop courses. Courseware distribution media include audio tape, video tape, CD-ROM, and 3.5" disks (PC). 


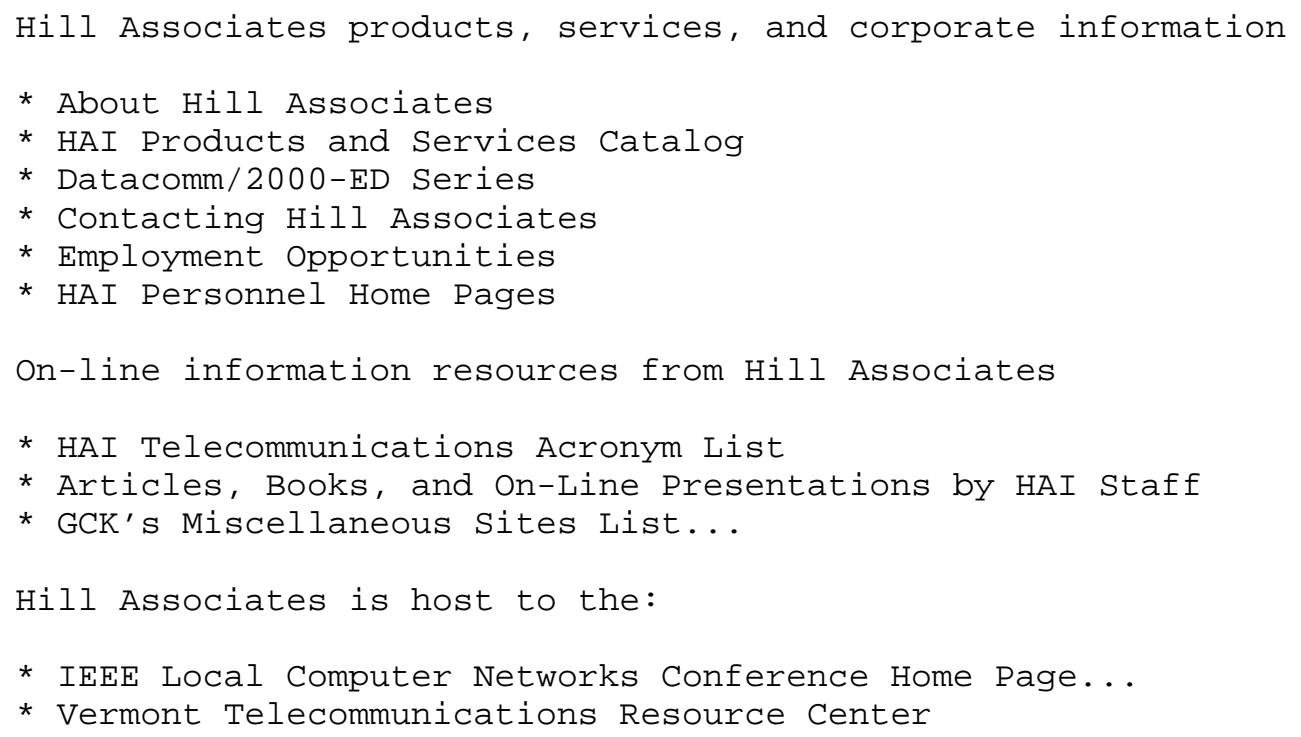




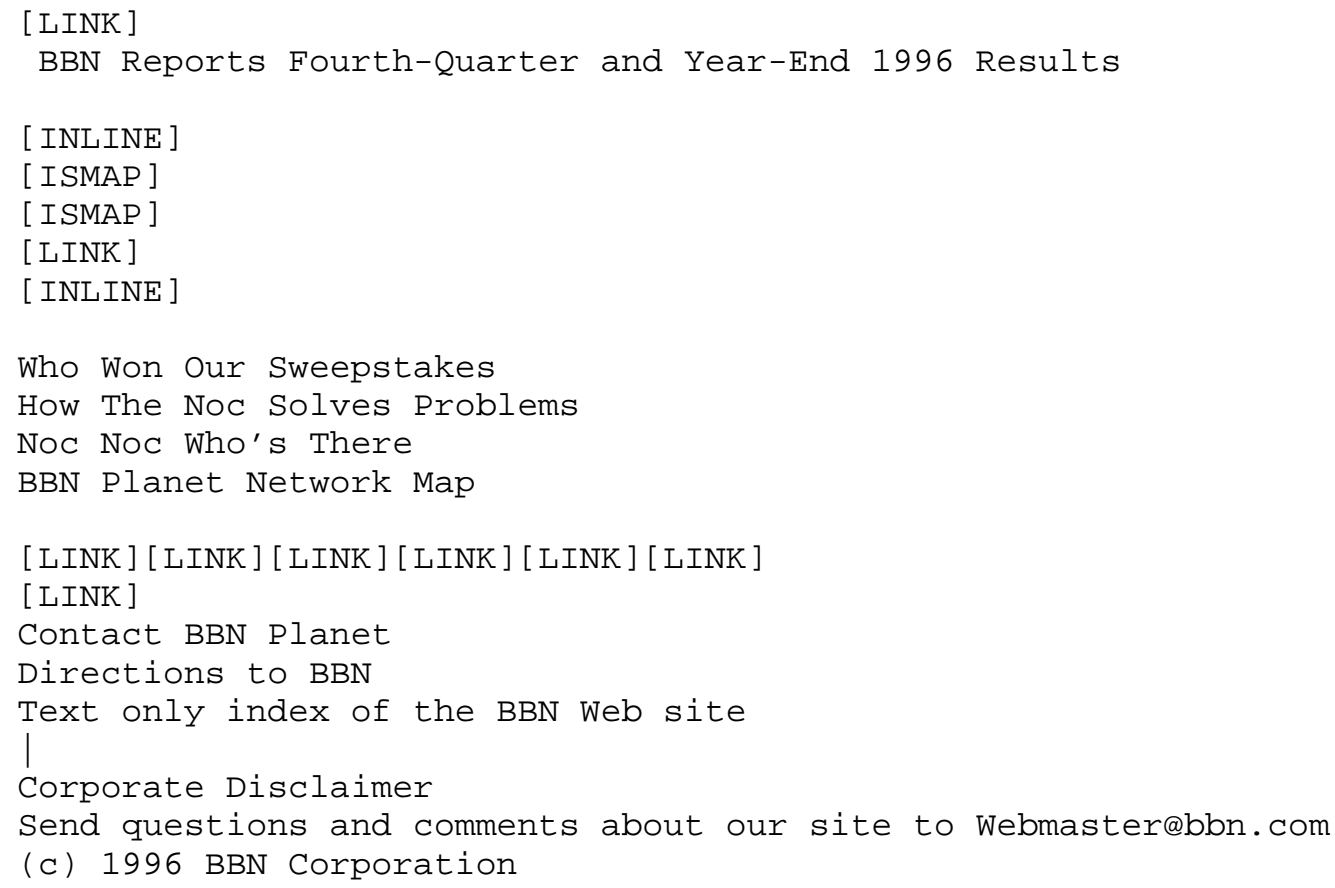

7.1. Uniform Resource Locators

As more and more protocols have become available to identify files, archive and server sites, news lists, and other information resources on the Internet, it was inevitable that some shorthand would arise to make it easier to designate these sources. The common shorthand format is called the Uniform Resource Locator. The list below provides information on how the URL format should be interpreted for the protocols and resources that will be discussed in this document. A complete description of the URL format may be found in [4] . 
file://host/directory/file-name

Identifies a specific file. E.g., the file htmlasst in the edu directory at host ftp.cs.da would be denoted, using the full URL form: <URL:file://ftp.cs.da/edu/htmlasst>.

ftp://user:passwordehost:port/directory/file-name

Identifies an FTP site. E.g.:

ftp://ftp.eff.org/pub/EFF/Policy/Crypto/*.

gopher://host:port/gopher-path

Identifies a Gopher site and menu path; a "00" at the start of the path indicates a directory and "11" indicates a file. E.g.: gopher://info.umd.edu:901/00/info/Government/Factbook92.

http://host:port/directory/file-name?searchpart

Identifies a WWW server location. E.g.:

http://info.isoc.org/home.html.

mailto:e-mail_address

Identifies an individual's Internet mail address. E.g.: mailto:s.shepardehill.com.

telnet://user:passwordehost:port/

Identifies a TELNET location (the trailing "/" is optional). E.g.: telnet://envnet:hennikerdenvnet.gsfc.nasa.gov.

\subsection{User Directories on the Web}

While finding users on the Internet remains somewhat like alchemy if using the tools and utilities mentioned earlier, the web has added a new dimension to finding people. Since 1995, many telephone companies have placed national white and yellow page telephone directories online, accessible via the World Wide Web.

For a while, it seemed that the easiest and most reliable approach to finding people's e-mail address on the Internet was to look up their telephone number on the Web, call them, and ask for their e-mail address! More recently, however, many third parties are augmenting the standard telephone directory with an e-mail directory. These services primarily rely on users voluntarily registering, resulting in incomplete databases because most users don't know about all of the services. Nevertheless, some of the personal directory services available via the Web with which e-mail addresses (and telephone numbers) can be found include Four11 Directory Services

(http://www.Four11.com/), Excite

(http://www.excite.com/Reference/locators.html), and Yahoo! People Search (http://www.yahoo.com/search/people/). 
In addition, the Knowbot Information Service (KIS), CNRI's automated username database search tool described earlier in this document, is also available on the Web, at http://info.cnri.reston.va.us/kis.html. Users can select several options for the KIS search, including the InterNIC, MILNET, MCImail, and Latin American Internic databases; UNIX finger and whois servers; and X.500 databases.

7.3. Other Service Accessible Via the Web

Many of the other utilities described earlier in this document can also be accessed via the WWW. In general, the Web browser acts as a viewer to a remote client rather than requiring specialized software on the user's system.

Several sites provide DNS information, obviating the need for a user to have a local DNS client such as NSLOOKUP. The hosts http://nsl.milepost.com/dns/ and http://sh1.ro.com/ mprevost/netutils/dig.html are among the best DNS sites, allowing the user to access all DNS information. The site http://www.bankes.com/nslookup.htm allows users to do multiple, sequential searches at a given domain. Other Web sites providing simple DNS name/address translation services include http://rhinoceros.cs.inf.shizuoka.ac.jp/dns.html, http://www.engin.umich.edu/htbin/DNSquery, http://www.lublin.pl/cgibin/ns/nsgate, and http://www.trytel.com/cgi-bin/weblookup.

Ping is another service available on the Web. The http://sh1.ro.com/ mprevost/netutils/ping.html page allows a user to select a host name, number of times to ping (1-10), and number of seconds between each ping (1-10), and returns a set of summary statistics. Other Web-based ping sites include http://www.net.cmu.edu/bin/ping (sends ten pings, and reports the times and min/max/avg summary statistics) and http://www.uia.ac.be/cc/ping.html (indicates whether the target host is alive or not).

Traceroute is also available on the Web. Unfortunately, these servers trace the route from their host to a host that the user chooses, rather than from the user's host to the target. Nevertheless, interesting route information can be found at http://www.net. cmu.edu/bin/traceroute. Traceroute service and a list of a number of other traceroute sites on the Web can be found at http://www.lublin.pl/cgi-bin/trace/traceroute. 
Access to archie is also available via the WWW, where your browser acts as the graphical interface to an archie server. To find a list of archie servers, and to access them via the Web, point your browser at http://www.yahoo.com/Computers_and_Internet/Internet/FTP_Sites/ Searching/Archie/.

Finally, even Finger can be found on the World Wide Web; check out http://sh1.ro.com/ mprevost/netutils/finger.html.

8. Discussion Lists and Newsgroups

Among the most useful features of the Internet are the discussion lists that have become available to allow individuals to discuss topics of mutual concern. Discussion list topics range from SCUBA diving and home brewing of beer to AIDS research and foreign policy. Several, naturally, deal specifically with the Internet, TCP/IP protocols, and the impact of new technologies.

Most of the discussion lists accessible from the Internet are unmoderated, meaning that anyone can send a message to the list's central repository and the message will then be automatically forwarded to all subscribers of the list. These lists provide very fast turn-around between submission of a message and delivery, but often result in a lot of messages (including inappropriate junk mail, or "spam"). A moderated list has an extra step; a human list moderator examines all messages before they are forwarded to ensure that the messages are appropriate to the list and not needlessly inflammatory!

Users should be warned that some lists generate a large number of messages each day. Before subscribing to too many lists, be sure that you are aware of local policies and/or charges governing access to discussion lists and e-mail storage.

\subsection{Internet Discussion Lists}

Mail can be sent to almost all Internet lists at an address with the following form:

list_name@host_name

The common convention when users want to subscribe, unsubscribe, or handle any other administrative matter is to send a message to the list administrator; do not send administrivia to the main list address! The list administrator can usually be found at:

list_name-REQUESTQhost_name 
To subscribe to a list, it is often enough to place the word "subscribe" in the main body of the message, although a line with the format:

subscribe list_name your_full_name

will satisfy most mail servers. A similar message may be used to get off a list; just use the word "unsubscribe" followed by the list name. Not every list follows this convention, but it is a safe bet if you don't have better information!

\subsection{LISTSERV}

A large set of discussion groups is maintained using a program called LISTSERV. LISTSERV is a service provided widely on BITNET and EARN, although it is also available to Internet users. A LISTSERV User Guide can be found on the Web at http://www.earn.net/lug/notice.html.

Mail can be sent to most LISTSERV lists at an address with the following form:

$$
\text { list_name@host_name }
$$

The common convention when users want to subscribe, unsubscribe, or handle any other administrative matter is to send commands in a message to the LISTSERV server; do not send administrivia to the main list address! The list server can usually be found at:

\section{LISTSERV@host_name}

LISTSERV commands are placed in the main body of e-mail messages sent to an appropriate list server location. Once you have found a list of interest, you can send a message to the appropriate address with any appropriate command, such as:

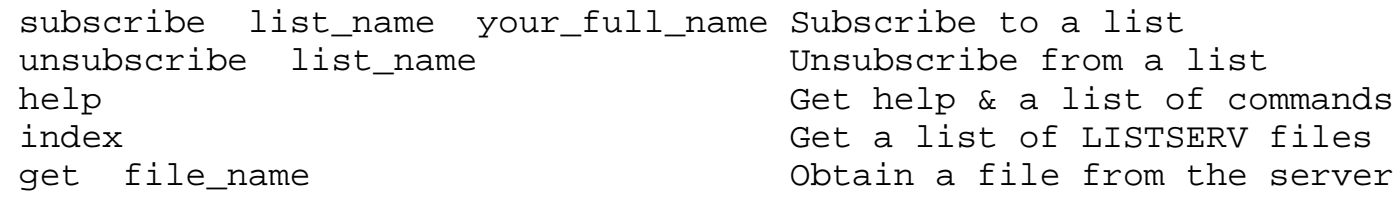

8.3. Majordomo

Majordomo is another popular list server for Internet discussion lists. The Web site http://www.greatcircle.com/majordomo/ has a large amount of information about Majordomo. 
Mail is sent to Majordomo lists using the same general address format as above:

list_name@host_name

The common convention when users want to subscribe, unsubscribe, or handle any other administrative matter is to send a message to the Majordomo list server; do not send administrivia to the main list address! The Majordomo server can usually be found at:

MAJORDOMO@host_name

Majordomo commands are placed in the main body of e-mail messages sent to an appropriate list server location. Available commands include:

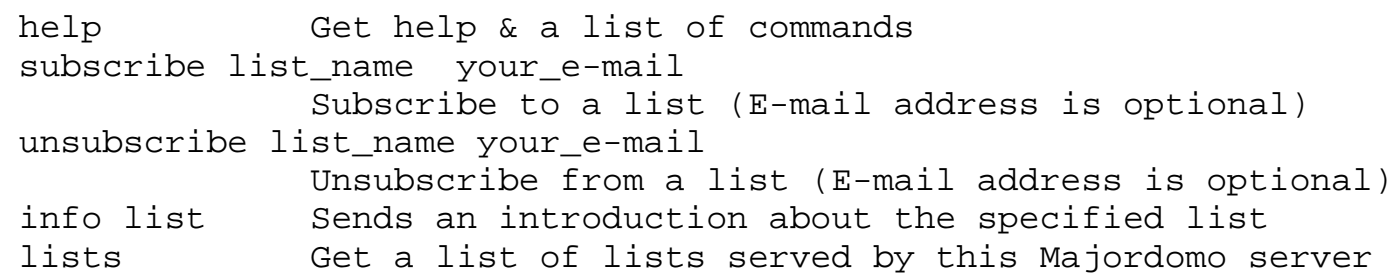

8.4. Usenet

Usenet, also known as NETNEWS or Usenet news, is another information source with its own set of special interest mailing lists organized into newsgroups. Usenet originated on UNIX systems but has migrated to many other types of hosts. Usenet clients, called newsreaders, use the Network News Transfer Protocol [13] and are available for virtually any operating system; several web browsers, in fact, have this capability built in.

While Usenet newsgroups are usually accessible at Internet sites, a prospective Usenet client host must have appropriate newsreader software to be able to read news. Users will have to check with their local host or network administrator to find out what usenet newsgroups are locally available, as well as the local policies for using them.

Usenet newsgroup names are hierarchical in nature. The first part of the name, called the hierarchy, provides an indication about the general subject area. There are two types of hierarchies, called mainstream and alternative; the total number of newsgroups is in the thousands. The news.announce.newusers newsgroup is a good place for new Usenet users to find a detailed introduction to the use of Usenet, as well as an introduction to its culture. 
Usenet mainstream hierarchies are established by a process that requires the approval of a majority of Usenet members. Most sites that receive a NETNEWS feed receive all of these hierarchies, which include:

$\begin{array}{ll}\text { comp } & \text { Computers } \\ \text { misc } & \text { Miscellaneous } \\ \text { news } & \text { Network news } \\ \text { rec } & \text { Recreation } \\ \text { sci } & \text { Science } \\ \text { soc } & \text { Social issues } \\ \text { talk } & \text { Various discussion lists }\end{array}$

The alternative hierarchies include lists that may be set up at any site that has the server software and disk space. These lists are not formally part of Usenet and, therefore, may not be received by all sites getting NETNEWS. The alternative hierarchies include:

$\begin{array}{ll}\text { alt } & \text { Alternate miscellaneous discussion lists } \\ \text { bionet } & \text { Biology, medicine, and life sciences } \\ \text { bit } & \text { BITNET discussion lists } \\ \text { biz } & \text { Various business-related discussion lists } \\ \text { ddn } & \text { Defense Data Network } \\ \text { gnu } & \text { GNU lists } \\ \text { ieee } & \text { IEEE information } \\ \text { info } & \text { Various Internet and other networking information } \\ \text { k12 } & \text { K-12 education } \\ \text { u3b } & \text { AT\&T 3B computers } \\ \text { vmsnet } & \text { Digital's VMS operating system }\end{array}$

8.5 Finding Discussion Lists and Newsgroups

Armed with the rules for signing up for a discussion list or accessing a newsgroup, how does one find an appropriate list given one's interests?

There are tens of thousands of e-mail discussion lists on the Internet. One List of Lists may be found using anonymous FTP at ftp://sri.com/netinfo/interest-groups.txt; the List of Lists can be searched using a Web browser by going to

http://catalog.com/vivian/interest-group-search.html. Other places to look are the Publicly Accessible Mailing Lists index at http://www.neosoft.com/internet/paml/byname.html and the LISZT Directory of E-Mail Discussion Groups at http://www.liszt.com. 
To obtain a list of LISTSERV lists, send e-mail to

listservabitnic.cren. net with the command lists global in the body of the message. Alternatively, look on the Web at http://www.tile.net/tile/listserv/index.html. The Web site http://www.liszt.com has a Mailing Lists Database of lists served by LISTSERV and Majordomo.

There are also thousands of Usenet newsgroups. One Usenet archive can be found at gopher://rtfm.mit.edu/11//pub/usenet/news.answers; see the /active-newsgroups and /alt-hierarchies subdirectories. Usenet news may also be read at gopher://gopher.bham.ac.uk/11/Usenet. A good Usenet search facility can be found at DejaNews at http://www.dejanews.com/; messages can also be posted to Usenet newsgroups from this site.

Note that there is often some overlap between Usenet newsgroups and Internet discussion lists. Some individuals join both lists in these circumstances or, often, there is cross-posting of messages. Some Usenet newsgroup discussions are forwarded onto an Internet mailing list by an individual site to provide access to those users who do not have Usenet available.

9. Internet Documentation

To fully appreciate and understand what is going on within the Internet community, users might wish to obtain the occasional Internet specification. The main body of Internet documents are Request for Comments (RFCs), although a variety of RFC subsets have been defined for various specific purposes. The sections below will describe the RFCs and other documentation, and how to get them.

The Internet standardization process is alluded to in the following sections. The Internet Engineering Task Force (IETF) is the guiding body for Internet standards; their Web site is http://www.ietf.org. The IETF operates under the auspices of the Internet society (ISOC), which has a Web site at http://www.isoc.org. For complete, up-to-date information on obtaining Internet documentation, go to the InterNIC's Web site at http://ds.internic.net/ds/dspg0intdoc.html. The IETF's history and role in the Internet today is described in Kessler [15]. For information on the organizations involved in the IETF standards process, see RFC 2028 [11]. For information on the relationship between the IETF and ISOC, see RFC 2031 [12].

\subsection{Request for Comments (RFCs)}

RFCs are the body of literature comprising Internet protocols, standards, research questions, hot topics, humor (especially those dated 1 April), and general information. Each RFC is uniquely issued 


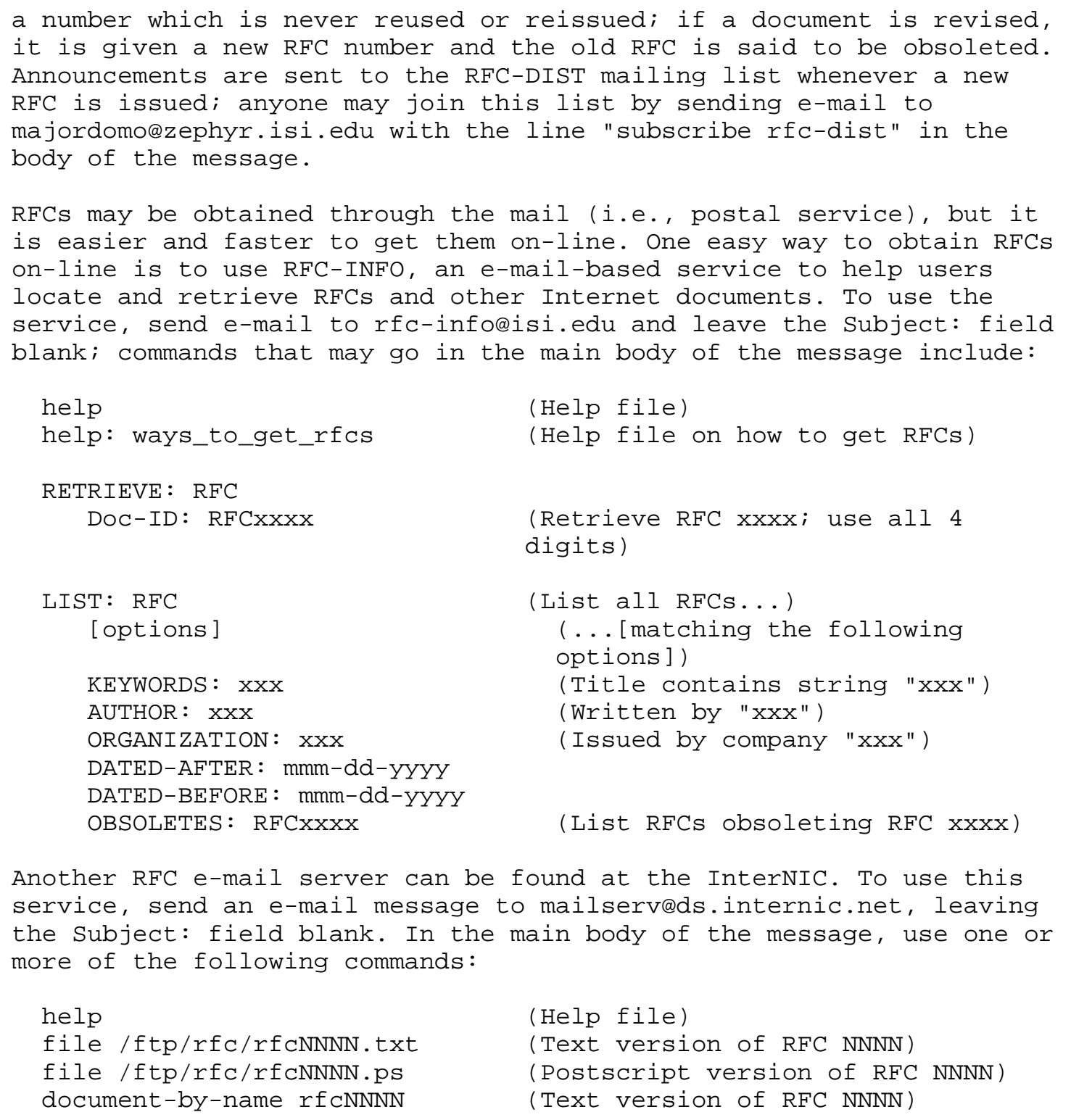


TABLE 1. Primary RFC Repositories.

$\begin{array}{ll}\text { HOST ADDRESS } & \text { DIRECTORY } \\ \text { ds.internic.net } & \text { rfc } \\ \text { nis.nsf.net } & \text { internet/documents/rfc } \\ \text { nisc.jvnc.net } & \text { rfc } \\ \text { ftp.isi.edu } & \text { in-notes } \\ \text { wuarchive.wustl.edu } & \text { info/rfc } \\ \text { src.doc.ic.ac.uk } & \text { rfc } \\ \text { ftp.ncren.net } & \text { rfc } \\ \text { ftp.sesqui.net } & \text { pub/rfc } \\ \text { nis.garr.it } & \text { mirrors/RFC } \\ \text { funet.fi } & \text { rfc } \\ \text { munnari.oz.au } & \text { rfc }\end{array}$

To obtain an RFC via anonymous FTP, connect to one of the RFC repositories listed in Table 1 using FTP. After connecting, change to the appropriate RFC directory (as shown in Table 1) using the cd command. To obtain a particular file, use the get command:

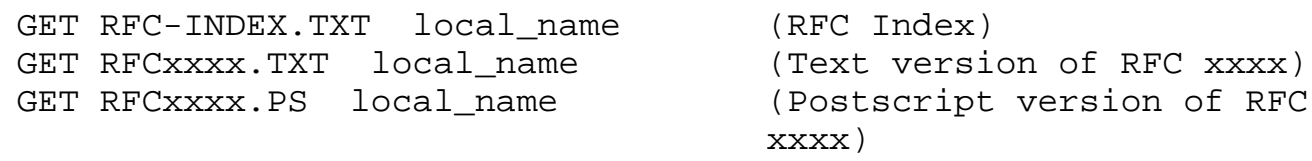

The RFC index, or a specific reference to an $\mathrm{RFC}$, will indicate whether the RFC is available in ASCII text (.txt) or Postscript (.ps) format. By convention, all RFCs are available in ASCII while some are also available in Postscript where use of graphics and/or different fonts adds more information or clarity; an increasing number are also being converted to HTML. Be aware that the index file is very large, containing the citing for over 2,000 documents. Note that not all RFCs numbered below 698 (July 1975) are available on-line.

Finally, the InterNIC's Web site at http://ds.internic.net/ds/dspglintdoc.html contains the RFC index and a complete set of RFCs. More information about Web-based RFC servers can be found at http://www.isi.edu/rfc-editor/rfc-sources.html.

The sample dialogue below, although highly abbreviated, shows a user obtaining RFC 1594 (Answers to Commonly asked "New Internet User" Questions) using e-mail and anonymous FTP. 


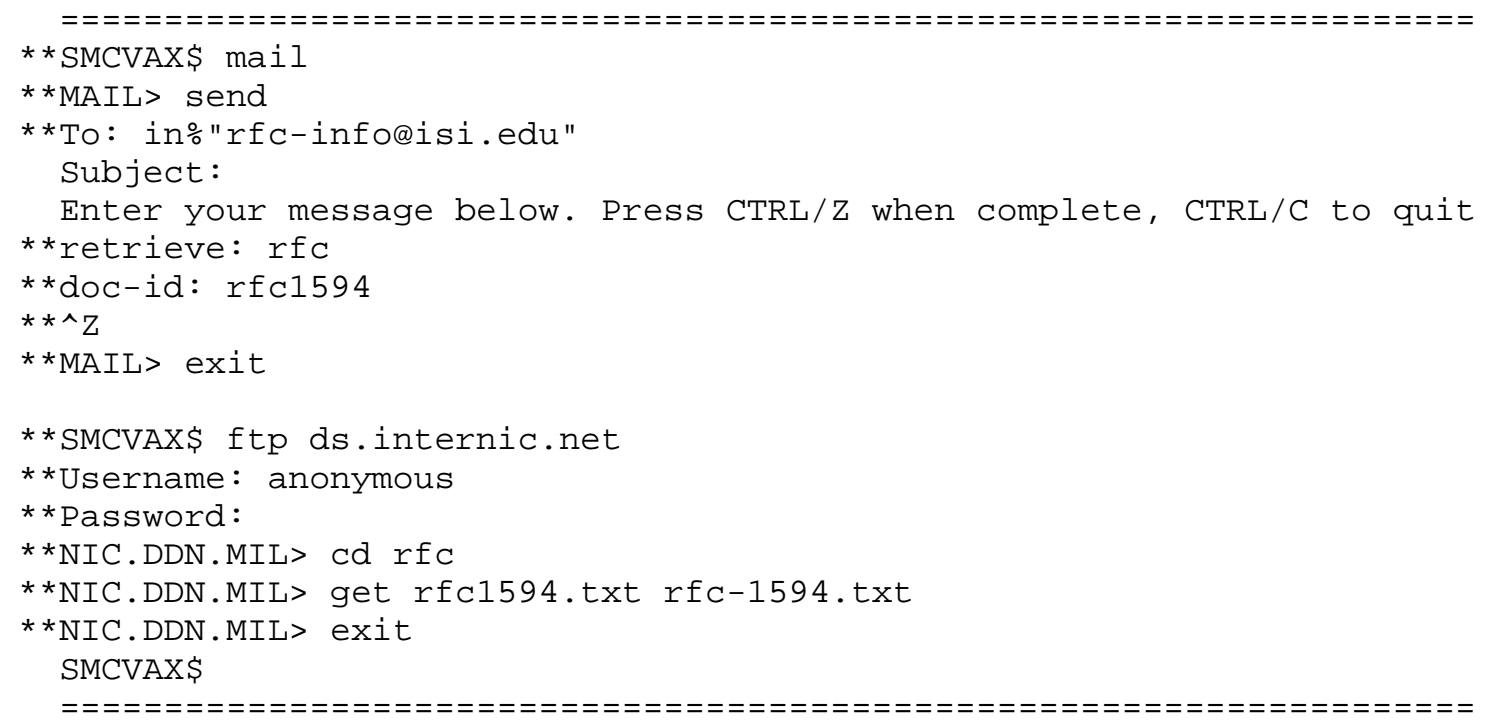

\subsection{Internet Standards}

RFCs describe many aspects of the Internet. By the early 1990s, however, so many specifications of various protocols had been written that it was not always clear as to which documents represented standards for the Internet. For that reason, a subset of RFCs have been designated as STDs to identify them as Internet standards.

Unlike RFC numbers that are never reused, STD numbers always refer to the latest version of the standard. UDP, for example, would be completely identified as "STD-6/RFC-768." Note that STD numbers refer to a standard, which is not necessarily a single document; STD 19, for example, is the NetBIOS Service Protocols standard comprising RFCs 1001 and 1002, and a complete citation for this standard would be "STD-19/RFC-001/RFC-1002."

The availability of new STDs is announced on the RFC-DIST mailing list. STD-1 [23] always refers to the latest list of "Internet Official Protocol standards". The Internet standards process is described in RFC 2026 [5] and STD notes are explained in RFC 1311 [24].

STDs can be obtained as RFCs via anonymous FTP from any RFC repository. In addition, some RFC sites (such as ds.internic.net) provide an STD directory so that STD documents can be found in the path /STD/XX.TXT, where $\mathrm{xx}$ refers to the STD number. 
STD documents may be obtained as RFCs using the methods described in Section 9.1. STDs may also be obtained via the RFC-INFO server using the RETRIEVE: STD and Doc-ID: STDXxxx commands. Also, check out the InterNIC's Web site at http://www.internic.net/std/ for the STD index and a complete set of STDs.

\subsection{For Your Information Documents}

The For Your Information (FYI) series of RFCs provides Internet users with information about many topics related to the Internet. FYI topics range from historical to explanatory to tutorial, and are aimed at the wide spectrum of people that use the Internet. The FYI series includes answers to frequently asked questions by both beginning and seasoned users of the Internet, an annotated bibliography of Internet books, and an explanation of the domain name system.

Like the STDs, an FYI number always refers to the latest version of an FYI. FYI 4, for example, refers to the answers to commonly asked questions by new Internet users; its complete citation would be "FYI-4/RFC-1594." The FYI notes are explained in FYI 1 [18].

FYIs can be obtained as RFCs via anonymous FTP from any RFC repository. In addition, some RFC sites (such as ds.internic.net) provide an FYI directory so that FYI documents can be found in the path /FYI/XX.TXT, where xx refers to the FYI number.

FYI documents may be obtained as RFCs using the methods described in Section 9.1. FYIs may also be obtained via the RFC-INFO server using the RETRIEVE: FYI and Doc-ID: FYIXXXX commands. Also, check out the InterNIC's Web site at http://www.internic.net/fyi/ for the FYI index and a complete set of FYIs.

\subsection{Best Current Practices}

Standards track RFCs are formally part of the IETF standards process, subject to peer review, and intended to culminate in an official Internet standard. Other RFCs are published on a less formal basis and are not part of the IETF process. To provide a mechanism of publishing relevant technical information which it endorsed, the IETF created a new series of RFCs, called the Best Current Practices (BCP) series. BCP topics include variances from the Internet standards process and IP address allocation in private networks. 
Like the STDs and FYIs, a BCP number always refers to the latest version of a BCP. BCP 5, for example, describes an IP address allocation plan for private networks; its complete citation would be "BCP-5/RFC-1918." The BCP process is explained in BCP 1 [25].

BCP documents may be obtained as RFCs using the methods described in Section 9.1. BCPS may also be obtained via the RFC-INFO server using the RETRIEVE: BCP and Doc-ID: BCPXxxx commands. Also, check out the RFC Editor's Web site at http://www.isi.edu/rfc-editor/ for the BCP index and a complete set of BCPs.

\subsection{RARE Technical Reports}

RARE, the Reseaux Associes pour la Recherche Europeenne (Association of European Research Networks), has a charter to promote and participate in the creation of a high-quality European computer communications infrastructure for the support of research endeavors. RARE member networks use Open Systems Interconnection (OSI) protocols and TCP/IP. To promote a closer relationship between RARE and the IETF, RARE Technical Reports (RTRs) have also been published as RFCs since the summer of 1993 .

RTR documents may be obtained as RFCs using the methods described in Section 9.1. RTRs may also be obtained via the RFC-INFO server using the RETRIEVE: RTR and Doc-ID: RTRxxxx commands. Also, check out the InterNIC's Web site at http://www.internic.net/rtr/ for the RTR index and a complete set of RTRs. Finally, RTRs may be obtained via anonymous FTP from ftp://ftp.rare.nl/rare/publications/rtr/.

10. Perusing the Internet

This guide is intended to provide the reader with a rudimentary ability to use the utilities that are provided by TCP/IP and the Internet. By now, it is clear that the user's knowledge, ability, and willingness to experiment are about the only limits to what can be accomplished.

There are several books that will help you get started finding sites on the Internet, including The INTERNET Yellow Pages [9]. But much more timely and up-to-date information can be found on the Internet itself, using such search tools as Yahoo! (http://www.yahoo.com), Excite (http://www.excite.com), Lycos (http://www.lycos.com), Webcrawler (http://www.webcrawler.com), and AltaAvista (http://altavista.digital.com). 
There are several other sources that cite locations from which to access specific information about a wide range of subjects using such tools as FTP, Telnet, Gopher, and WWW. One of the best periodic lists, and archives, is through the scout Report, a weekly publication by the InterNIC's Net Scout Services Project at the University of Wisconsin's Computer Science Department. To receive the Scout Report by e-mail each week, join the mailing list by sending email to listservelists.internic.net; place the line subscribe scout-report your_full_name in the body of the message to receive the text version or use subscribe scout-report-html your_full_name to receive the report in HTML. The scout Report is also available on the Web at http://www.cs.wisc.edu/scout/report and http://rs.internic.net/scout/report, or via anonymous FTP at ftp://rs.internic.net/scout/.

Another list is Yanoff's Internet Services List, which may be found at http://www.spectracom.com/islist/ or ftp://ftp.csd.uwm.edu/pub/inet.services.txt. Gary Kessler, one of the co-author's of this document, maintains his own eclectic Miscellaneous Sites List at http://www.together.net/ kessler/gck_site.html.

If you are looking for Internet-specific information, one good starting point is http://www.yahoo.com/Computers_and_Internet/Internet/. The InterNIC is another valuable resource, with their scout Report and Scout Toolkit (http://rs.internic.net/scout/toolkit).

There is also a fair amount of rudimentary tutorial information available on the Internet. The InterNIC cosponsors "The 15 Minute Series" (http://rs.internic.net/nic-support/15min/), a collection of free, modular, and extensible training materials on specific Internet topics. ROADMAP 96 (http://www.ua.edu/ crispen/roadmap.html) is a free, 27-lesson Internet training workshop over e-mail.

More books and specialized articles came out about the Internet in 1993 and 1994 than in all previous years (squared!), and that trend has seemed to continue into 1995, 1996, and beyond. Three books are worth notable mention because they do not directly relate to finding your way around, or finding things on, the Internet. Hafner and Lyon [8] have written Where Wizards Stay Up Late: The Origins of the Internet, a history of the development of the Advanced Research Projects Agency (ARPA), packet switching, and the ARPANET, focusing primarily on the $1960 \mathrm{~s}$ and 1970s. While culminating with the APRANET's 25th Anniversary in 1994, its main thrusts are on the groups building the ARPANET backbone (largely BBN) and the host-tohost application and communication protocols (largely the Network Working Group). Salus' book, Casting The Net: From ARPANET to 
INTERNET and beyond... [28], goes into the development of the network from the perspective of the people, protocols, applications, and networks. Including a set of "diversions," his book is a bit more whimsical than Hafner \& Lyon's. Finally, Carl Malamud has written a delightful book called Exploring the Internet: A Technical Travelogue [17], chronicling not the history of the Internet as much as a subset of the people currently active in building and defining it. This book will not teach you how to perform an anonymous FTP file transfer nor how to use Gopher, but provides insights about our network (and Carl's gastro-pathology) that no mere statistics can convey.

11. Acronyms and Abbreviations

$\begin{array}{ll}\text { ASCII } & \text { American Standard Code for Information Interchange } \\ \text { BCP } & \text { Best Current Practices } \\ \text { BITNET } & \text { Because It's Time Network } \\ \text { DDN } & \text { Defense Data Network } \\ \text { DNS } & \text { Domain Name System } \\ \text { EARN } & \text { European Academic Research Network } \\ \text { FAQ } & \text { Frequently Asked Questions list } \\ \text { FTP } & \text { File Transfer Protocol } \\ \text { FYI } & \text { For Your Information series of RFCs } \\ \text { HTML } & \text { Hypertext Markup Language } \\ \text { HTTP } & \text { Hypertext Transport Protocol } \\ \text { ICMP } & \text { Internet Control Message Protocol } \\ \text { IP } & \text { Internet Protocol } \\ \text { ISO } & \text { International Organization for Standardization } \\ \text { NetBIOS } & \text { Network Basic Input/Output System } \\ \text { NIC } & \text { Network Information Center } \\ \text { NICNAME } & \text { Network Information Center name service } \\ \text { NSF } & \text { National Science Foundation } \\ \text { NSFNET } & \text { National Science Foundation Network } \\ \text { RFC } & \text { Request For Comments } \\ \text { RARE } & \text { Reseaux Associes pour la Recherche Europeenne } \\ \text { RTR } & \text { RARE Technical Reports } \\ \text { STD } & \text { Internet Standards series of RFCs } \\ \text { TCP } & \text { Transmission Control Protocol } \\ \text { TTL } & \text { Time-To-Live } \\ \text { UDP } & \text { User Datagram Protocol } \\ \text { URL } & \text { Uniform Resource Locator } \\ \text { WAIS } & \text { Wide Area Information Server } \\ \text { WWW } & \text { World Wide Web } \\ & \end{array}$




\section{Security Considerations}

Security issues are not discussed in this memo.

\section{Acknowledgments}

Our thanks are given to all sites that we accessed or otherwise used system resources in preparation for this document. We also appreciate the comments and suggestions from our students and members of the Internet community, particularly after the last version of this document was circulated, including Mark Delany and the rest of the gang at the Australian Public Access Network Association, Margaret Hall (BBN), John Martin (RARE), Tom Maufer (3Com), Carol Monaghan (Hill Associates), Michael Patton (BBN), N. Todd Pritsky (Hill Associates), and Brian Williams. Special thanks are due to Joyce Reynolds for her continued encouragement and direction.

14. References

[1] Anklesaria, F., M. McCahill, P. Lindner, D. Johnson, D. Torrey, and B. Alberti, "The Internet Gopher Protocol," RFC 1436, University of Minnesota, March 1993.

[2] Berners-Lee, T. and D. Connolly, "Hypertext Markup Language - 2.0," RFC 1866, MIT/W3C, November 1995.

[3] , R. Fielding, and H. Frystyk, "Hypertext Transfer Protocol HTTP/1.0," RFC 1945, MIT/LCS, UC Irvine, MIT/LCS, May 1996.

[4] , L. Masinter, and M. McCahill, Editors, "Uniform Resource Locators (URL)," RFC 1738, CERN, Xerox Corp., University of Minnesota, December 1994.

[5] Bradner, S. "The Internet Standards Process -- Revision 3," RFC 2026, Harvard University, October 1996.

[6] Comer, D. Internetworking with TCP/IP, Vol. I: Principles, Protocols, and Architecture, 3/e. Englewood Cliffs (NJ) : PrenticeHall, 1995 .

[7] Feit, S. TCP/IP: Architecture, Protocols, and Implementation with IPv6 and IP Security, 2/e. New York: McGraw-Hill, 1997.

[8] Hafner, K. and M. Lyon. Where Wizards Stay Up Late: The Origins of the Internet. New York: Simon \& Schuster, 1997. 
[9] Hahn, H. and R. Stout. The Internet Yellow Pages, 3/e. Berkeley (CA) : Osborne McGraw-Hill, 1996.

[10] Harrenstien, K., M. Stahl, and E. Feinler, "NICNAME/WHOIS," RFC 954, SRI, October 1985.

[11] Hovey, R. and S. Bradner. "The Organizations Involved in the IETF Standards Process," RFC 2028, Digital, Harvard University, October 1996.

[12] Huizer, E. "IETF-ISOC Relationship," RFC 2031, SEC, October 1996.

[13] Kantor, B. and P. Lapsley. "Network News Transfer Protocol," RFC 977, U.C. San Diego, U.C. Berkeley, February 1986.

[14] Kessler, G.C. "An Overview of TCP/IP Protocols and the Internet." URL: http://www.hill.com/library/tcpip.html. Last accessed: 17 February 1997

[15] "IETF-History, Background, and Role in Today's Internet." URL: http://www.hill.com/library/ietf_hx.html. Last accessed: 17 February 1997 .

[16] "Running Your Own DNS." Network VAR, July 1996. (See also URL: http://www.hill.com/library/dns.html. Last accessed: 17 February 1997.)

[17] Malamud, C. Exploring the Internet: A Technical Travelogue. Englewood Cliffs (NJ): PTR Prentice Hall, 1992.

[18] Malkin, G.S. and J.K. Reynolds, "F.Y.I. on F.Y.I.: Introduction to the F.Y.I. notes," FYI 1/RFC 1150, Proteon, USC/Information Sciences Institute, March 1990.

[19] Mockapetris, P., "Domain Names - Concepts and Facilities," STD 13/RFC 1034, USC/Information Sciences Institute, November 1987.

[20] National Center for Supercomputer Applications (NCSA). "A Beginner's Guide to HTML." URL: http://wWW.ncsa.uiuc.edu/General/Internet/WWW/HTMLPrimer.html. Last accessed: 2 February 1997.

[21] Postel, J., "Domain Name System Structure and Delegation," USC/Information Sciences Institute, RFC 1591, March 1994.

[22] "Internet Control Message Protocol," USC/Information Sciences Institute, RFC 792, September 1981. 
[23] , Editor, "Internet Official Protocol Standards," STD 1/RFC 2000, Internet Architecture Board, February 1997.

[24] Sciences Institute, March 1992.

[25] _ T. Li, and Y. Rekhter, "Best Current Practices," BCP 1/RFC 1818, USC/Information Sciences Institute, Cisco Systems, August 1995.

[26] and J. Reynolds, "File Transfer Protocol (FTP)," STD 9/RFC 959, USC/Information Sciences Institute, October 1985. and J. Reynolds, "TELNET Protocol Specification,"

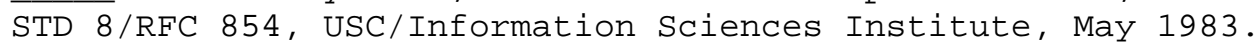

[28] Salus, P.H. Casting The Net: From ARPANET to INTERNET and beyond... Reading (MA): Addison-Wesley, 1995.

[29] Socolofsky, T.J. and C.J. Kale, "TCP/IP Tutorial," RFC 1180, Spider Systems Ltd., January 1991.

[30] Stevens, W.R. TCP/IP Illustrated, Volume 1: The Protocols. Reading (MA) : Addison-Wesley, 1994.

[31] Williamson, S., "Transition and Modernization of the Internet Registration Service," RFC 1400, Network Solutions, Inc., March 1993.

[32] Zimmerman, D., "The Finger User Information Protocol," RFC 1288, Rutgers University, December 1991.

15. Authors' Address

Gary C. Kessler

Hill Associates

17 Roosevelt Highway

Colchester, VT 05446

Phone: +1 802-655-8659

Fax: +1 802-655-7974

E-mail: kumquat@hill.com 
Steven D. Shepard

Hill Associates

17 Roosevelt Highway

Colchester, VT 05446

Phone: +1 802-655-8646

Fax: +1 802-655-7974

E-mail: s.shepardehill.com 